\title{
Title: Cytoplasmic protein aggregates interfere with nucleo-cytoplasmic transport of protein and RNA
}

\author{
Authors: Andreas C. Woerner ${ }^{1}$, Frédéric Frottin ${ }^{1}$, Daniel Hornburg ${ }^{2}$, Li R. Feng ${ }^{1, \dagger}$, Felix \\ Meissner $^{2}$, Maria Patra ${ }^{3}$, Jörg Tatzelt ${ }^{3,4}$, Matthias Mann²,6, Konstanze F. Winklhofer ${ }^{3,5,6}$, F. \\ Ulrich Hartl ${ }^{1,6 *}$, and Mark S. Hipp ${ }^{1,6 *}$
}

\begin{abstract}
Affiliations:
${ }^{1}$ Departments of Cellular Biochemistry and ${ }^{2}$ Proteomics and Signal Transduction, Max Planck Institute of Biochemistry, Am Klopferspitz 18, D-82152 Martinsried, Germany.

${ }^{3}$ Neurobiochemistry, Adolf Butenandt Institute, Ludwig Maximilians University, Schillerstr. 44, D-80336 Munich, Germany

${ }^{4}$ Departments of Biochemistry of Neurodegenerative Diseases and ${ }^{5}$ Molecular Cell Biology, Institute of Biochemistry and Pathobiochemistry, Ruhr University Bochum, Universitätsstraße 150, D-44801 Bochum, Germany.

${ }^{6}$ Munich Cluster for Systems Neurology (SyNergy), D-80336 Munich, Germany.

$\dagger$ Present address: National Institutes of Health, Bethesda, MD, 20892, USA.

* Corresponding author. E-mail: hipp@,biochem.mpg.de (M.S.H.); uhartl@biochem.mpg.de (F.U.H.)
\end{abstract}




\begin{abstract}
Amyloid-like protein aggregation is associated with neurodegeneration and other pathologies. The nature of the toxic aggregate species and their mechanism of action remain elusive. Here we analyzed the compartment-specificity of aggregate toxicity using artificial $\beta$ sheet proteins as well as fragments of mutant huntingtin and TDP-43. Aggregation in the cytoplasm interfered with nucleo-cytoplasmic protein and RNA transport. In contrast, the same proteins did not inhibit transport when forming inclusions in the nucleus at or around the nucleolus. Protein aggregation in the cytoplasm, but not the nucleus, caused the sequestration and mislocalization of proteins containing disordered and low complexity sequences, including multiple factors of the nuclear import and export machinery. Our results suggest that impairment of nucleo-cytoplasmic transport contributes to the cellular pathology of various aggregate deposition diseases.
\end{abstract}

One Sentence Summary: Protein aggregates in the cytoplasm, but not in the nucleus, inhibit transport of proteins and mRNA across the nuclear envelope.

Main Text: Cellular protein homeostasis (proteostasis) is controlled by a complex network of factors, including molecular chaperones, proteases, and their regulators $(1,2)$. Misfolded proteins are recognized and either refolded, degraded, or sequestered to distinct cellular sites. However, when these proteostasis machineries become compromised, as is increasingly the case during aging $(1,3)$, aberrant proteins tend to accumulate as toxic aggregate species. This process is associated with numerous neurodegenerative diseases and other disorders (4). 
Intracellular protein aggregation in disease occurs predominantly in the cytoplasm and nucleus, with toxic effects possibly arising in both locations (5-7). Here we investigated the basic mechanisms by which aggregates exert cytotoxicity in a compartment-specific manner. We used authentic disease proteins and artificial $\beta$-sheet proteins known to form prefibrillar and fibrillar aggregates $(8,9)$. The artificial proteins are members of a combinatorial library designed to form $\beta$-strands. They have no evolved biological function and their mRNA does not contain tri- or hexanucleotide repeat regions (fig. S1A to C), which may contribute to neurodegenerative pathology $(10,11)$. We analyzed two of these proteins, $\beta 17$ and $\beta 23$, which vary in aggregation efficiency and toxicity. An artificial protein forming a soluble $\alpha$-helical bundle ( $\alpha \mathrm{S} 824)$ served as a non-toxic control $(8,9)$.

$\beta 17$ and $\beta 23$ form aggregates in the cytoplasm and nucleus of HEK293T cells (9). To identify the cellular compartment in which toxicity arises, we added a nuclear export sequence (NES) or nuclear localization sequence (NLS) to the proteins (fig. S1A). NES- $\beta 17$ and NES- $\beta 23$ formed large cytoplasmic inclusions that stained with the amyloid-specific dye NIAD-4 (Fig. 1A and fig. S2A), suggesting the presence of cross- $\beta$ structure $(12,13)$. NLS- $\beta 17$ and NLS- $\beta 23$ formed multiple nuclear inclusions in close proximity to the nucleolus (Fig. 1A and B). These inclusions stained only weakly with NIAD-4 and displayed reduced detergent solubility compared to the cytoplasmic aggregates (Fig. 1C and fig. S2A). The $\beta$-proteins in both cytoplasmic and nuclear inclusions were highly immobile, as measured with $\beta 17$-GFP fusion proteins (fig. S2B).

The toxicity of the nuclear $\beta$-proteins was significantly reduced compared to their cytoplasmic counterparts and the proteins lacking a localization signal (Fig. 1D and fig. S3A and B). This reduced toxicity was not due to lower expression levels (Fig. 1C and fig. S3C) or the 
presence of an NLS. Point mutations in the NLS prevented nuclear targeting and increased toxicity (fig. S3A and B). Thus, otherwise virtually identical proteins form biochemically distinct aggregate species in the cytoplasm and nucleus that differ markedly in toxicity.

The nuclear aggregates co-immunoprecipitated with the abundant, negatively charged nucleolar protein nucleophosmin-1 (NPM1) (Fig. 1E)(14). During mitosis, when the nuclear envelope breaks down and nucleoli are disassembled, NPM1 distributed diffusely throughout the cell, but maintained its apparent association with the aggregates, as shown for NLS- $\beta 17$ (fig. S4). Thus, NPM1 may have a chaperone-like function in shielding the surfaces of potentially toxic aggregates (15), and this may have prevented access of the NIAD-4 dye. Note that cells with cytoplasmic aggregates underwent mitosis only very rarely.

The expression of $\beta$-proteins in the cytoplasm altered the distribution of nuclear pore complex (NPC) proteins and partially dislocated NPC proteins to the cytoplasmic inclusions of NES- $\beta 17$ and NES- $\beta 23$ (Fig. 1A). In contrast, expression of NLS- $\beta 17$ and NLS- $\beta 23$ had no visible effect on NPC integrity (Fig. 1A and fig. S3A). To test whether the cytoplasmic aggregates interfered with nuclear transport processes, we employed a GFP reporter carrying NES and NLS signals (Shuttle-GFP). In control cells, S-GFP accumulated predominantly in the cytoplasm (Fig. 2 A and B). Expression of NLS- $\beta 17$ did not alter the distribution of S-GFP. However, NES- $\beta 17$ caused a significant retention of S-GFP in the nucleus (Fig. $2 \mathrm{~A}$ and B). This inhibition of nuclear export was even more pronounced in cells containing cytoplasmic inclusions of polyQ-expanded Htt exon 1 (Htt96Q) (Fig. 2 A and B). Upon addition of the exportin inhibitor Leptomycin B (LMB) (16) to control cells, S-GFP accumulated in the nucleus within 15 min (Fig. $2 \mathrm{~A}$ and B), reflecting rapid nuclear import. Again this import was significantly inhibited by cytoplasmic aggregates of NES- $\beta 17$ and Htt96Q, but not by nuclear 
NLS- $\beta 17$. Thus, the cytoplasmic aggregates analyzed interfere with both import and export of proteins through the nuclear pore.

We also analyzed the nuclear translocation of the endogenous protein NF- $\mathrm{kB}$ subunit $\mathrm{p} 65$ upon activation with the cytokine TNF $\alpha$ (17). p65 readily translocated in control cells and in cells expressing nuclear $\beta$-sheet proteins. In contrast, cells containing cytoplasmic aggregates failed to support p65 transport (Fig. 2C), even though phosphorylation of p65 and degradation of IкB were not altered (fig. S5).

Next we tested whether cytoplasmic aggregates also affected the transport of RNA. In control cells, poly(A) RNA (mRNA) was distributed throughout the cytoplasm and was present in small nuclear ribonucleic particles (Fig. 3A and (18)). Expression of the nuclear $\beta$-proteins did not influence this pattern. However, we observed a substantial nuclear accumulation of mRNA in cells expressing either the cytoplasmic $\beta$-proteins, Htt96Q, or a GFP-tagged C-terminal fragment of TAR DNA binding protein-43 (TDP-F4) (Fig. 3A and B and fig. S6A). Similar fragments of TDP-43 aggregate in the cytoplasm of neuronal cells in amyotrophic lateral sclerosis and frontotemporal dementia (19).

Impairment of mRNA export from the nucleus would explain the reported reduction in protein synthesis capacity of $\beta$-protein expressing cells (9). Indeed, expression of the cytoplasmic $\beta$-proteins, but not the nuclear $\beta$-proteins, resulted in a significant reduction of protein biosynthesis (Fig. 3C and fig. S6B). Quantitative analysis of mRNA levels confirmed that the strong reduction in cytoplasmic mRNA content was due to inhibition of export from the nucleus and not to reduced RNA synthesis or increased turnover (fig. S6C and D).

Inhibition of mRNA export by cytoplasmic aggregates was reproduced in neuroblastoma cells and primary neurons (fig. S7 and S8). Note that mutant Htt preferentially forms nuclear 
inclusions in primary neurons (7) and these did not interfere with mRNA export (fig. S8). However, in the striatum and cortex of the R6/2 mouse, which is transgenic for human polyQexpanded Htt exon 1 (20), mutant $\mathrm{Htt}$ forms cytoplasmic and nuclear aggregates (21). We observed significant alterations in mRNA distribution in brain slices of 9-week-old R6/2 mice. More than $10 \%$ of the neurons analyzed showed either an accumulation of mRNA in the nucleus or an overall reduction in mRNA levels (Fig. 3D and E and fig. S9A).

Protein aggregates may exert toxicity through aberrant interactions with other proteins, resulting in their functional impairment and sequestration $(9,22)$. Quantitative interactome analysis of the $\beta$-proteins in HEK cells previously identified several proteins involved in nuclear transport, including importin subunit alpha-1/KPNA2 and THOC2 (9), a subunit of the THO (suppressor of the transcriptional defects of $\underline{h} p r 1 \Delta$ by overexpression) complex involved in mRNA export $(23,24)$. A similar interactome analysis in primary neurons expressing either $\beta 17$ GFP or GFP alone identified the seven-subunit THO complex as a highly enriched $\beta$-protein interactor (Fig. 4A and Table S1 to 3). Indeed, expression of NES- $\beta 17$ caused mislocalization and in some cases aggregation of THOC proteins in the cytoplasm, as shown using THOC2 antibodies (Fig. 4B). Remarkably, the nuclear inclusions of NLS- $\beta 17$ did not co-aggregate with THOC2, although the majority of THOC2 was present in the nucleus. Mislocalization of THOC2 to the cytoplasm also occurred in cells containing cytoplasmic Htt96Q or TDP-F4 aggregates (Fig. 4B), and in the brains of R6/2 mice (fig. S9B). THOC2 often formed separate inclusions that did not co-localize with these aggregates (Fig. 4B), consistent with the prion-like behavior described for certain RNA binding proteins when dislocated to the cytoplasm (25). Importin $\alpha-1$ and importin $\alpha-3$ were also mislocalized to cytoplasmic $\beta$-protein aggregates (fig. S10A and B). 
Besides THOC, the $\beta$-protein interactome in primary neurons contained splicing factors and several other RNA binding proteins (Fig 4A and Table S1), suggesting that the cytoplasmic aggregates not only affect mRNA export, but also nuclear mRNA processing. Indeed, cells containing cytoplasmic aggregates exhibited a more pronounced accumulation of mRNA in the nucleus than cells in which THOC2 was down-regulated (fig. S10C) (26).

Our findings provide insight into common mechanisms that are likely to contribute to aggregate toxicity in neurodegenerative diseases and other disorders. Cytoplasmic aggregates of artificial $\beta$-sheet proteins and authentic disease proteins caused a pronounced impairment of nucleo-cytoplasmic transport, and a redistribution of nuclear shuttle factors to the cytosol. Several of these transport proteins, including THOC2 (fig. S11), contain disordered and low complexity sequences that may render them vulnerable to interactions with the interactive surfaces of cytoplasmic aggregates (Table S2). Thus the inhibition of nuclear transport observed in this system can be assigned to proteotoxicity, rather than to aberrant interactions between RNA repeat sequences and RNA binding proteins (10). Such repeat RNAs occur in the coding region or in untranslated regions of disease genes and are associated with amyotrophic lateral sclerosis, frontotemporal dementia and CAG repeat disorders (11). Their co-existence with protein aggregates has confounded the analysis of toxicity mechanisms $(10,27,28)$.

Surprisingly, otherwise identical aggregation-prone proteins did not interfere with nucleo-cytoplasmic transport when directed to the nucleus. How the nuclear environment alters the interaction properties of the $\beta$-proteins remains to be investigated in detail, but our findings suggest that the negatively charged, nucleolar protein NPM1 is involved in shielding interactive aggregate surfaces. Indeed, recent reports that misfolded cytoplasmic proteins are actively imported into the nucleus for degradation (29) support a protective role for the intranuclear 
sequestration of aberrant proteins (7). However, specific aggregation-prone proteins may escape recognition by the nuclear quality control machinery. For example, nuclear aggregates of polyQ expansion proteins have been shown to interfere with transcriptional regulation by engaging transcription factors containing glutamine repeats (30). A better understanding of nuclear proteostasis may help in developing new strategies for the treatment of proteinopathies. 


\section{References and Notes:}

1. E. T. Powers, R. I. Morimoto, A. Dillin, J. W. Kelly, W. E. Balch, Biological and chemical approaches to diseases of proteostasis deficiency. Annu Rev Biochem 78, 959 (2009).

2. F. U. Hartl, A. Bracher, M. Hayer-Hartl, Molecular chaperones in protein folding and proteostasis. Nature 475, 324 (Jul 21, 2011).

3. R. C. Taylor, A. Dillin, Aging as an event of proteostasis collapse. Cold Spring Harb Perspect Biol 3, (May, 2011).

4. T. P. Knowles, M. Vendruscolo, C. M. Dobson, The amyloid state and its association with protein misfolding diseases. Nat Rev Mol Cell Biol 15, 384 (Jun, 2014).

5. K. Y. Liu et al., Disruption of the nuclear membrane by perinuclear inclusions of mutant huntingtin causes cell-cycle re-entry and striatal cell death in mouse and cell models of Huntington's disease. Hum Mol Genet 24, 1602 (Mar 15, 2015).

6. A. S. Hackam, R. Singaraja, T. Zhang, L. Gan, M. R. Hayden, In vitro evidence for both the nucleus and cytoplasm as subcellular sites of pathogenesis in Huntington's disease. Hum Mol Genet 8, 25 (Jan, 1999).

7. F. Saudou, S. Finkbeiner, D. Devys, M. E. Greenberg, Huntingtin acts in the nucleus to induce apoptosis but death does not correlate with the formation of intranuclear inclusions. Cell 95, 55 (Oct 2, 1998).

8. M. W. West et al., De novo amyloid proteins from designed combinatorial libraries. Proc Natl Acad Sci U $S A$ 96, 11211 (Sep 28, 1999).

9. H. Olzscha et al., Amyloid-like aggregates sequester numerous metastable proteins with essential cellular functions. Cell 144, 67 (Jan 7, 2011).

10. K. Zhang et al., The C9orf72 repeat expansion disrupts nucleocytoplasmic transport. Nature 525, 56 (Sep $3,2015)$.

11. R. Nalavade, N. Griesche, D. P. Ryan, S. Hildebrand, S. Krauss, Mechanisms of RNA-induced toxicity in CAG repeat disorders. Cell death \& disease 4, e752 (2013).

12. E. E. Nesterov et al., In vivo optical imaging of amyloid aggregates in brain: design of fluorescent markers. Angew Chem Int Ed Engl 44, 5452 (Aug 26, 2005).

13. T. Jakhria et al., beta2-microglobulin amyloid fibrils are nanoparticles that disrupt lysosomal membrane protein trafficking and inhibit protein degradation by lysosomes. J Biol Chem 289, 35781 (Dec 26, 2014).

14. W. Y. Chan et al., Characterization of the cDNA encoding human nucleophosmin and studies of its role in normal and abnormal growth. Biochemistry 28, 1033 (Feb 7, 1989).

15. A. Szebeni, M. O. Olson, Nucleolar protein B23 has molecular chaperone activities. Protein Sci 8, 905 (Apr, 1999).

16. B. Wolff, J. J. Sanglier, Y. Wang, Leptomycin B is an inhibitor of nuclear export: inhibition of nucleocytoplasmic translocation of the human immunodeficiency virus type 1 (HIV-1) Rev protein and Revdependent mRNA. Chem Biol 4, 139 (Feb, 1997).

17. P. A. Baeuerle, D. Baltimore, Activation of DNA-binding activity in an apparently cytoplasmic precursor of the NF-kappa B transcription factor. Cell 53, 211 (Apr 22, 1988).

18. K. C. Carter, K. L. Taneja, J. B. Lawrence, Discrete nuclear domains of poly(A) RNA and their relationship to the functional organization of the nucleus. J Cell Biol 115, 1191 (Dec, 1991).

19. C. Yang et al., The C-terminal TDP-43 fragments have a high aggregation propensity and harm neurons by a dominant-negative mechanism. PLoS One 5, e15878 (2010).

20. L. Mangiarini et al., Exon 1 of the HD gene with an expanded CAG repeat is sufficient to cause a progressive neurological phenotype in transgenic mice. Cell 87, 493 (Nov 1, 1996).

21. H. Li et al., Ultrastructural localization and progressive formation of neuropil aggregates in Huntington's disease transgenic mice. Hum Mol Genet 8, 1227 (Jul, 1999).

22. B. Bolognesi et al., ANS binding reveals common features of cytotoxic amyloid species. ACS Chem Biol 5 , 735 (Aug 20, 2010).

23. N. Visa et al., A pre-mRNA-binding protein accompanies the RNA from the gene through the nuclear pores and into polysomes. Cell 84, 253 (Jan 26, 1996).

24. H. Cheng et al., Human mRNA export machinery recruited to the 5' end of mRNA. Cell 127, 1389 (Dec 29, 2006).

25. O. D. King, A. D. Gitler, J. Shorter, The tip of the iceberg: RNA-binding proteins with prion-like domains in neurodegenerative disease. Brain Res 1462, 61 (Jun 26, 2012). 
26. B. Chi et al., Aly and THO are required for assembly of the human TREX complex and association of TREX components with the spliced mRNA. Nucleic Acids Res 41, 1294 (Jan, 2013).

27. B. D. Freibaum et al., GGGGCC repeat expansion in C9orf72 compromises nucleocytoplasmic transport. Nature 525, 129 (Sep 3, 2015).

28. A. Jovicic et al., Modifiers of C9orf72 dipeptide repeat toxicity connect nucleocytoplasmic transport defects to FTD/ALS. Nat Neurosci 18, 1226 (Aug 26, 2015).

29. M. S. Hipp, S. H. Park, F. U. Hartl, Proteostasis impairment in protein-misfolding and -aggregation diseases. Trends Cell Biol 24, 506 (Sep, 2014).

30. K. L. Sugars, D. C. Rubinsztein, Transcriptional abnormalities in Huntington disease. Trends Genet 19, 233 (May, 2003).

31. G. Schaffar et al., Cellular toxicity of polyglutamine expansion proteins: mechanism of transcription factor deactivation. Mol Cell 15, 95 (Jul 2, 2004).

32. H. C. Chang, C. M. Kaiser, F. U. Hartl, J. M. Barral, De novo folding of GFP fusion proteins: high efficiency in eukaryotes but not in bacteria. J Mol Biol 353, 397 (Oct 21, 2005).

33. M. S. Shearman, S. R. Hawtin, V. J. Tailor, The intracellular component of cellular 3-(4,5-dimethylthiazol2-yl)-2, 5-diphenyltetrazolium bromide (MTT) reduction is specifically inhibited by beta-amyloid peptides. J Neurochem 65, 218 (Jul, 1995).

34. J. A. Jablonski, M. Caputi, Role of cellular RNA processing factors in human immunodeficiency virus type 1 mRNA metabolism, replication, and infectivity. J Virol 83, 981 (Jan, 2009).

35. J. Rappsilber, M. Mann, Y. Ishihama, Protocol for micro-purification, enrichment, pre-fractionation and storage of peptides for proteomics using StageTips. Nature protocols 2, 1896 (2007).

36. A. Michalski et al., Ultra high resolution linear ion trap Orbitrap mass spectrometer (Orbitrap Elite) facilitates top down LC MS/MS and versatile peptide fragmentation modes. Mol Cell Proteomics 11, O111 013698 (Mar, 2012).

37. J. Cox, M. Mann, MaxQuant enables high peptide identification rates, individualized p.p.b.-range mass accuracies and proteome-wide protein quantification. Nat Biotechnol 26, 1367 (Dec, 2008).

38. J. Cox et al., Andromeda: a peptide search engine integrated into the MaxQuant environment. J Proteome Res 10, 1794 (Apr 1, 2011).

39. D. Hornburg et al., Deep proteomic evaluation of primary and cell line motoneuron disease models delineates major differences in neuronal characteristics. Mol Cell Proteomics 13, 3410 (Dec, 2014).

40. J. Cox et al., Accurate proteome-wide label-free quantification by delayed normalization and maximal peptide ratio extraction, termed MaxLFQ. Mol Cell Proteomics 13, 2513 (Sep, 2014).

41. R. C. Team. (2014).

42. V. G. Tusher, R. Tibshirani, G. Chu, Significance analysis of microarrays applied to the ionizing radiation response. Proc Natl Acad Sci US A 98, 5116 (Apr 24, 2001).

43. J. C. Wootton, Non-globular domains in protein sequences: automated segmentation using complexity measures. Computers \& chemistry 18, 269 (Sep, 1994).

44. M. E. Oates et al., D(2)P(2): database of disordered protein predictions. Nucleic Acids Res 41, D508 (Jan, 2013).

45. H. Wickham, The Split-Apply-Combine Strategy for Data Analysis. J Stat Softw 40, 1 (Apr, 2011).

46. Z. Dosztanyi, V. Csizmok, P. Tompa, I. Simon, IUPred: web server for the prediction of intrinsically unstructured regions of proteins based on estimated energy content. Bioinformatics 21, 3433 (Aug 15, 2005).

47. H. Wickham, ggplot2 Elegant Graphics for Data Analysis Introduction. Use R, 1 (2009). 
Acknowledgments: We thank R. Klein, K. Schulz-Trieglaff, and I. Dudanova from the Max Planck Institute of Neurobiology for help with the preparation of primary neurons and brain slices, and J. Cox, F. Hosp and M. Duerrbaum for support with proteomic data analysis. We thank C. Klaips and D. Balchin for critically reading the manuscript. The research leading to these results has received funding from the European Commission under Grant FP7 GA ERC-2012-SyG_318987-ToPAG (to A.C.W., D.H., M.M., F.U.H., and M.S.H.), the Munich Cluster for Systems Neurology (K.F.W, M.M, F.U.H. and M.S.H.), the German Research Foundation (J.T., K.F.W.), and the Hans and Ilse Breuer Foundation (M.P.). Data from the mass spectrometry analysis described in this manuscript can be found in the supplementary materials. 
Fig. 1. Targeting aggregation-prone proteins to cytoplasm or nucleus modulates their properties. HEK293T cells $24 \mathrm{~h}$ after transfection of the indicated NES- and NLS-proteins. (A) Anti-Myc (red), anti-NPC (nuclear pore complex) proteins (green) and DAPI (blue). Scale bar, $10 \mu \mathrm{m}$. (B) Anti-Myc (red), nucleoli labeled with anti-NPM1 antibodies (green) and DAPI (blue). Scale bar, $10 \mu \mathrm{m}$. (C) Solubility of $\beta$-proteins directed to cytoplasm and nucleus. S, soluble fraction; P, pellet fraction; H3, histone H3. (D) Viability of HEK293T cells expressing $\alpha \mathrm{S} 824$ and $\beta$-proteins, as measured by MTT assay. All values relative to untransfected control cells. Data are mean + standard deviation (SD), $N=4 .^{*}, \mathrm{p} \leq 0.05, * * *, \mathrm{p} \leq 0.001$ by unpaired Student's $t$-test. (E) HEK293T cells were transfected with the indicated constructs; following cell lysis, anti-Myc antibodies were used for immunoprecipitation (IP). T, total.

Fig. 2. Cytoplasmic aggregates interfere with nuclear protein transport. (A) HEK293T cells co-transfected with S-GFP (green) and either empty vector (Control), NES- $\beta 17$, NLS- $\beta 17$, or Htt96Q (red); DAPI (blue). Leptomycin B (LMB; $10 \mathrm{ng} / \mathrm{mL}$ ) was added for $15 \mathrm{~min}$ where indicated. Scale bar, $10 \mu \mathrm{m}$. (B) Quantification of S-GFP distribution from data in (A). X-axis shows enrichment of S-GFP in the nucleus relative to the cytoplasm. Data are mean $+\mathrm{SD}, \mathrm{N}=3$. *, $\mathrm{p} \leq 0.05, * *, \mathrm{p} \leq 0.01, * * *, \mathrm{p} \leq 0.001$ from unpaired Student's $t$-test. (C) HEK293T cells transfected with empty vector (Control), NES- $\beta 17$, or NLS- $\beta 17$ (red) were analyzed for NF- $\kappa \mathrm{B}$ p65 localization (green) with and without TNF $\alpha$ treatment (30 min). Nuclear DNA (blue). Scale bar, $10 \mu \mathrm{m}$.

Fig. 3. Protein aggregates in the cytoplasm inhibit mRNA export. (A) HEK293T cells were analyzed for polyA RNA (green), NES- $\beta 17$, NLS- $\beta 17$, Htt96Q or TDP-F4 (red); DAPI (blue). Scale bar, $10 \mu \mathrm{m}$. (B) Quantification of (A). Data are mean $+\mathrm{SD}, \mathrm{N}=3$. (C) Quantification of protein biosynthesis from data in fig. S6B. ${ }^{35} \mathrm{~S}-\mathrm{Met}$ incorporation in control cells was set to $100 \%$. Data are mean $+\mathrm{SD}, \mathrm{N}=3 .{ }^{*}, \mathrm{p} \leq 0.05, * *, \mathrm{p} \leq 0.01$ from unpaired Student's $t$-test. (D) Distribution of polyA RNA (green) in brain slices of 9-week-old wild-type (Wt) and R6/2 mice, transgenic for human polyQ-expanded Htt exon 1. PolyQ expanded Htt (red); nuclear DNA (blue). The middle panel shows an example of a cell with nuclear accumulation of mRNA and mRNA remaining in the cytoplasm. The right panel shows a cell with strongly reduced overall mRNA levels. (E) Quantification of fraction of cells from Wt and R6/2 mice with abnormal polyA RNA distribution from data in (D). At least 1,000 cells were analyzed per animal. Data are mean $+\mathrm{SD}, \mathrm{N}=3 . * *, \mathrm{p} \leq 0.01$ from unpaired Student's $t$-test.

Fig. 4. Cytoplasmic aggregates cause mislocalization of nuclear transport factors. (A) The $\beta 17-G F P$ interactome was determined by anti-GFP pull-down and quantitative mass spectrometry, using cells expressing GFP alone as a control. Significant interactors were analyzed for highly enriched annotations. The y-axis indicates the fold enrichment of annotations, the $\mathrm{x}$-axis depicts the significance of the respective enrichment. The THO complex was the most strongly enriched interactor of $\beta 17$-GFP in primary neurons. (B) Immunofluorescence analysis of HEK293T cells transfected with empty vector (Control), NES- 
$\beta 17$, NLS- $\beta 17$, Htt96Q, or TDP-F4. THOC2 (green), protein aggregates (red), DAPI (blue). Scale bar, $10 \mu \mathrm{m}$. 
Supplementary Materials:

Materials and Methods

Figures S1-S11

Tables S1-S3

References (31-47) 
Woerner et al. Fig.1

A
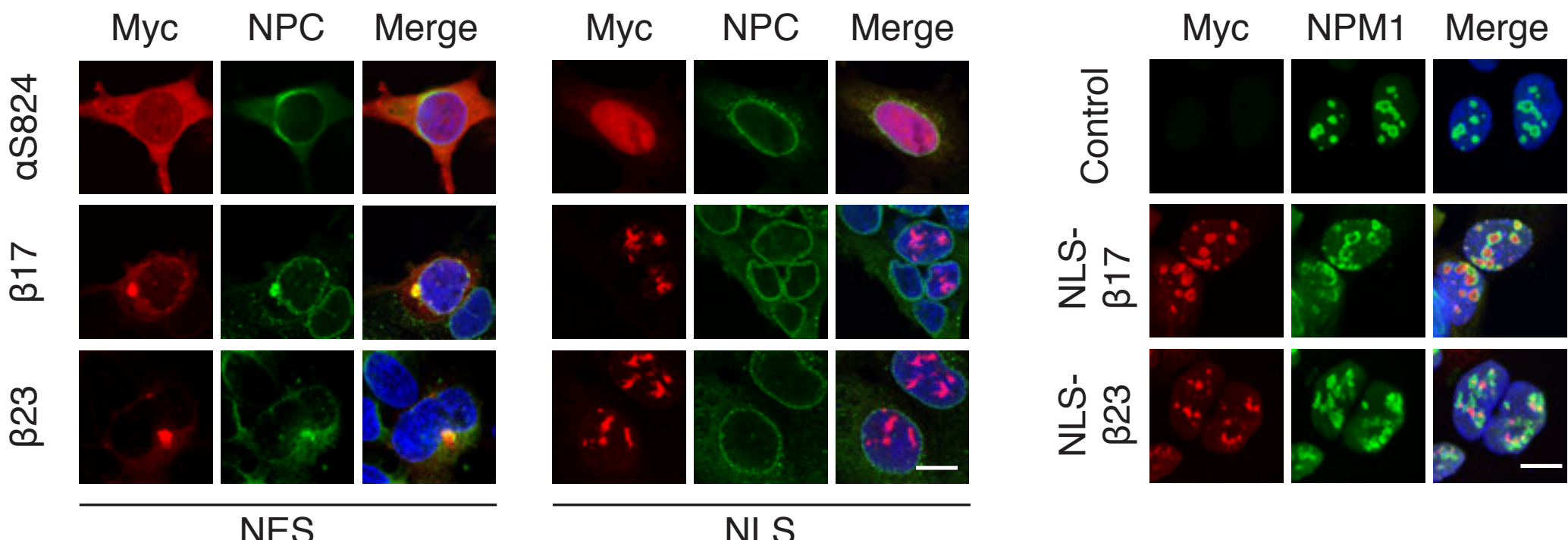

C

D

NES- NLS-
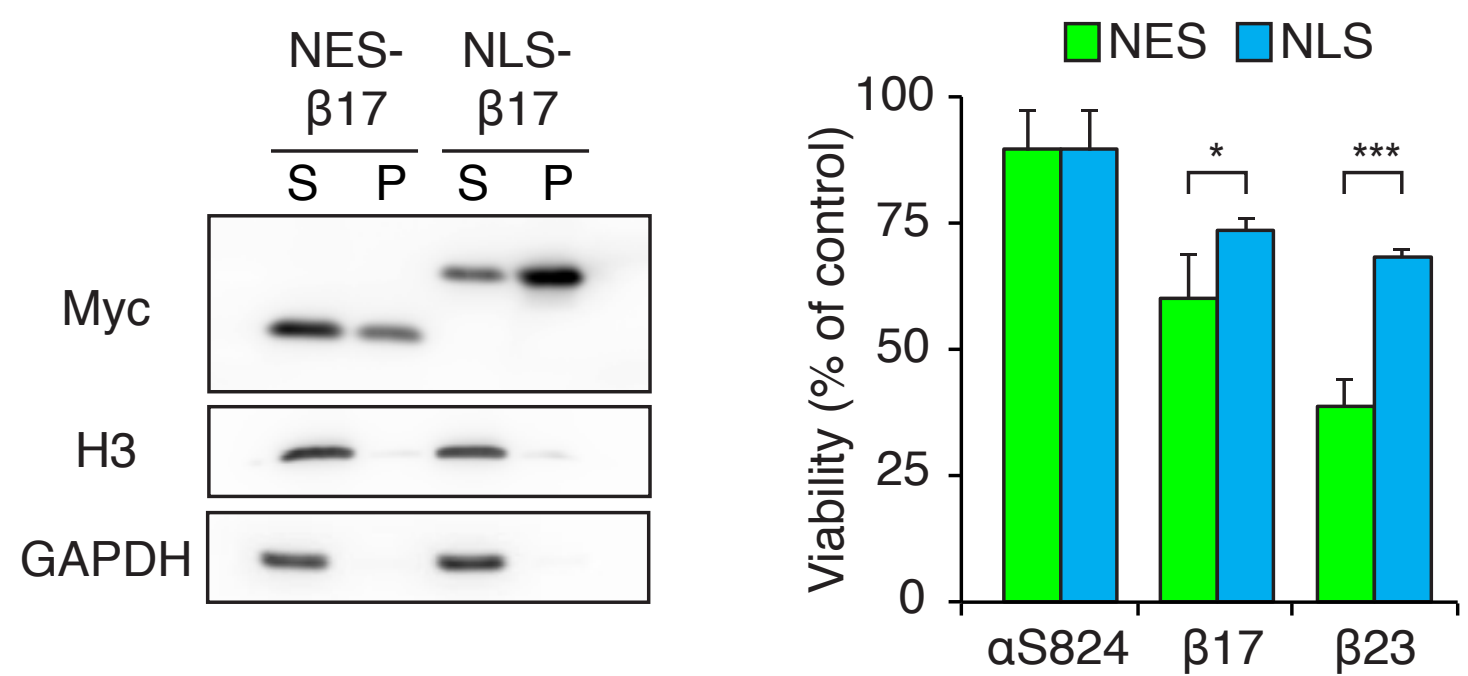

E

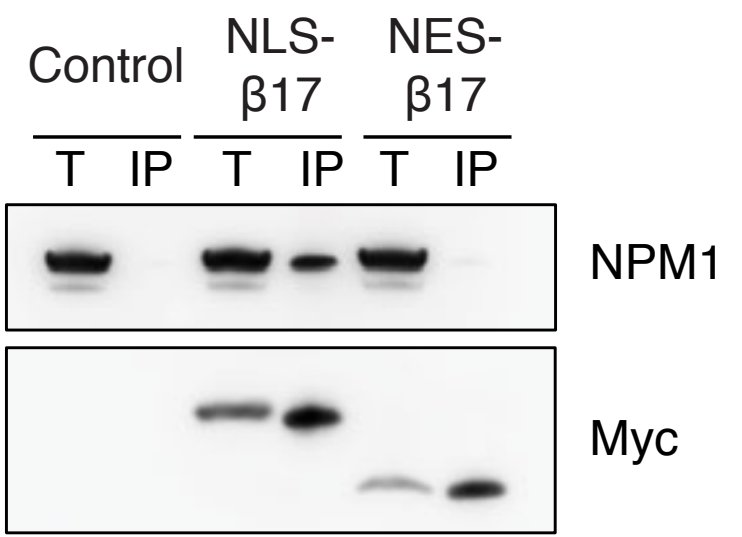


A

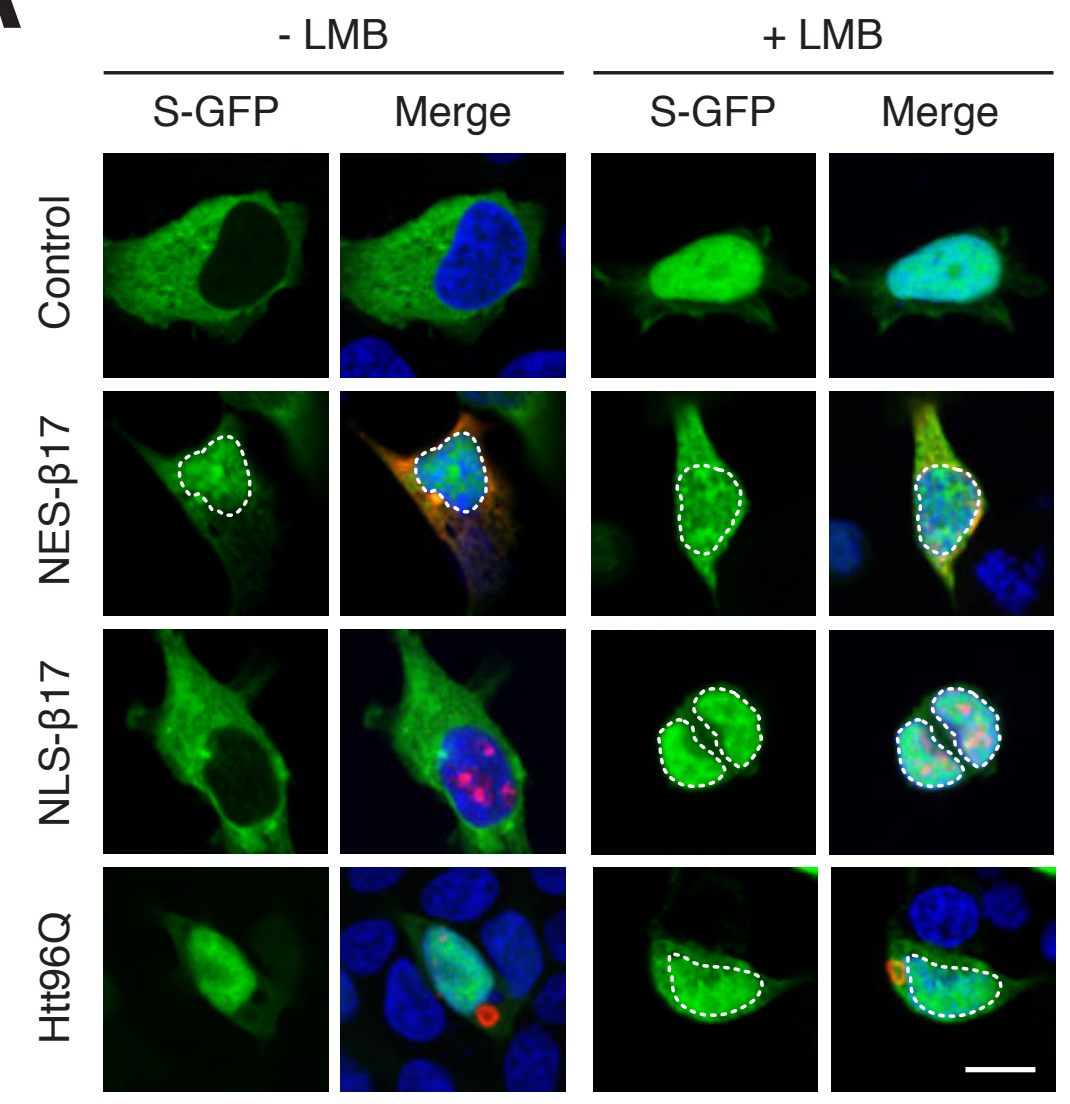

B S-GFP [Cytoplasm] : [Nucleus]

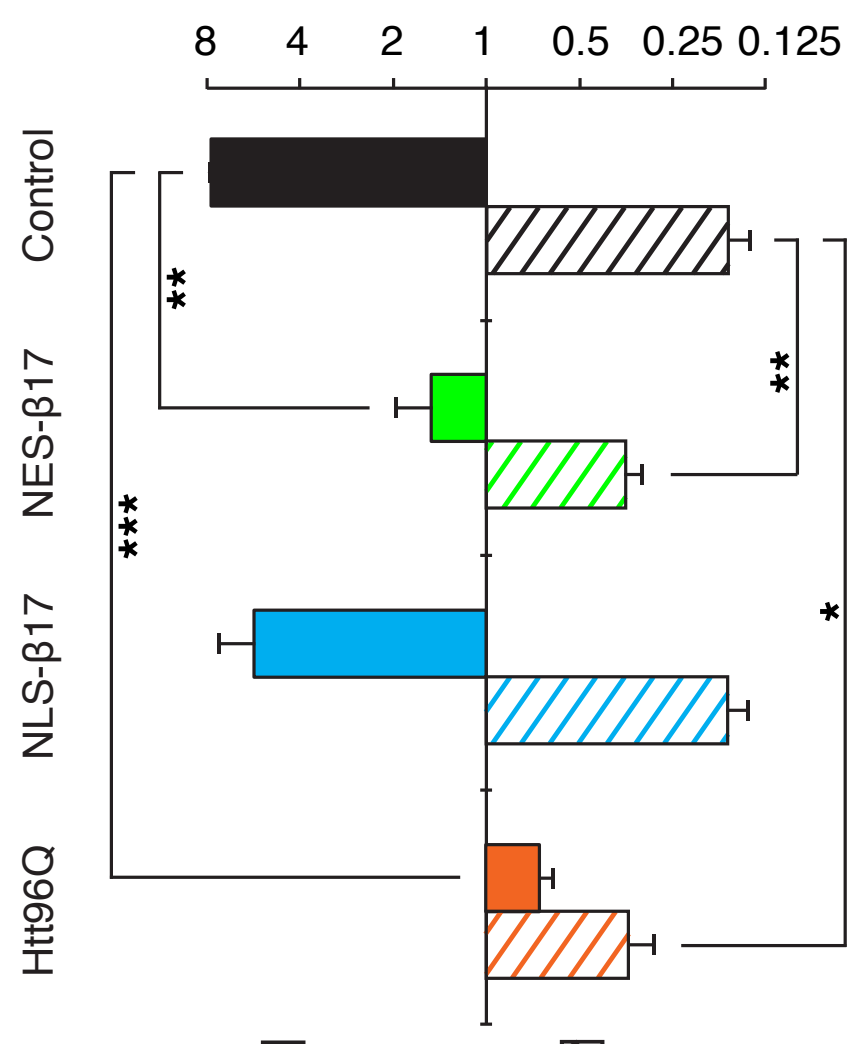

- LMB
C

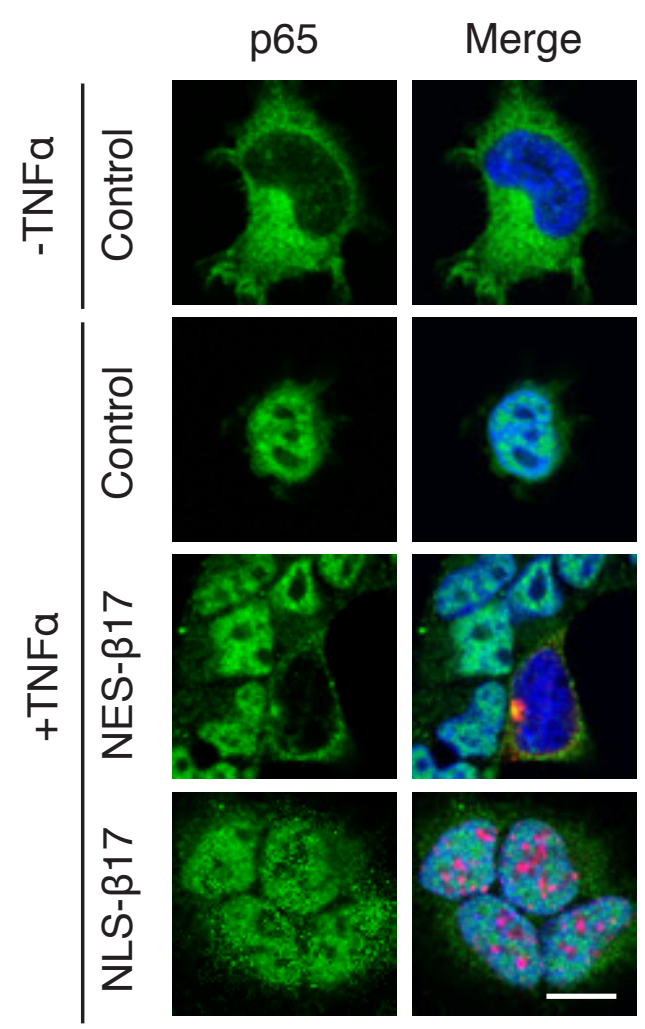


Woerner et al. Fig.3

A

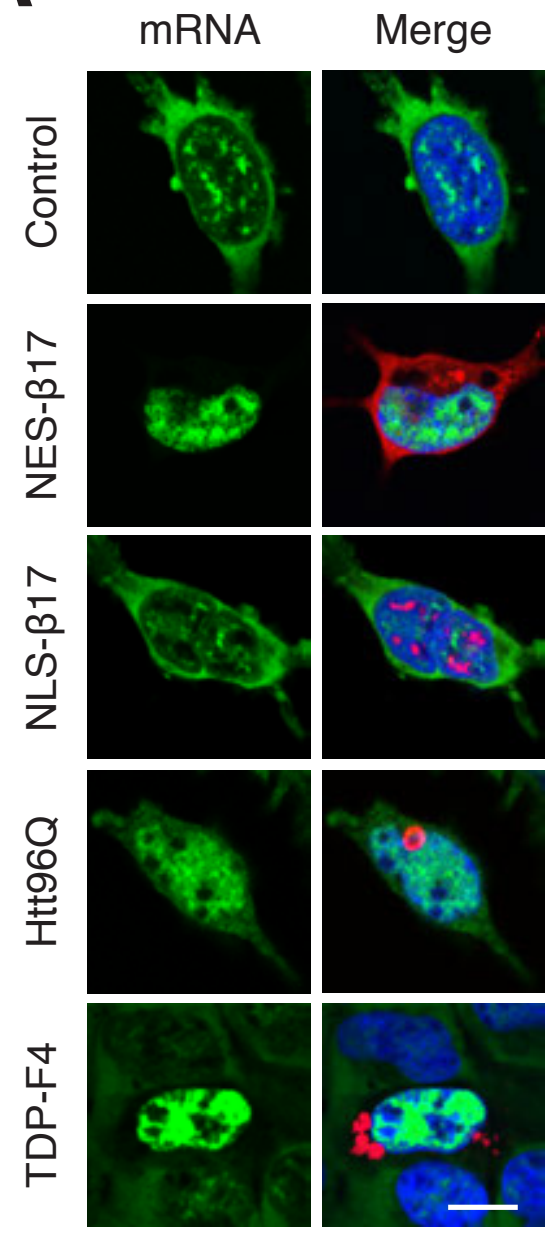

B

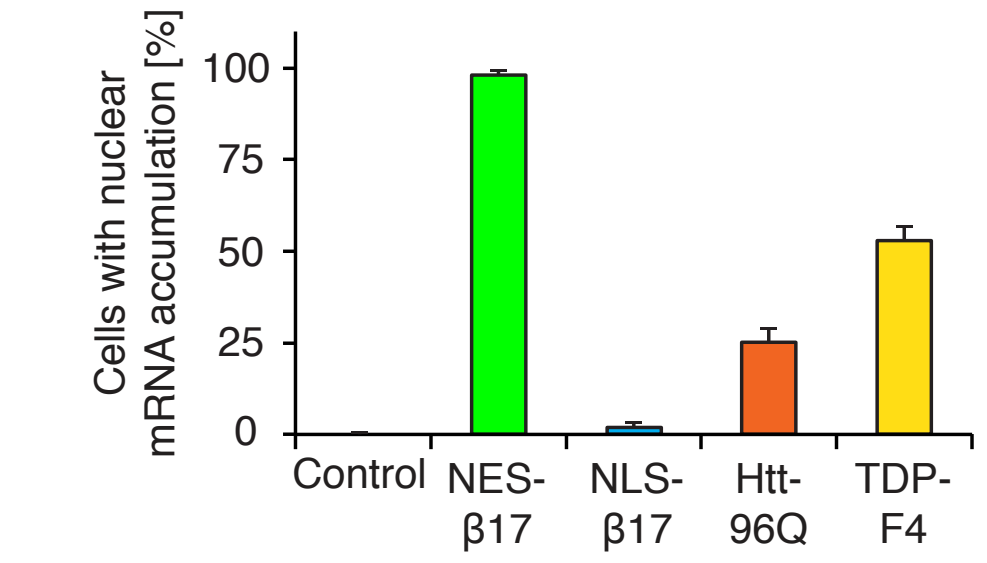

C

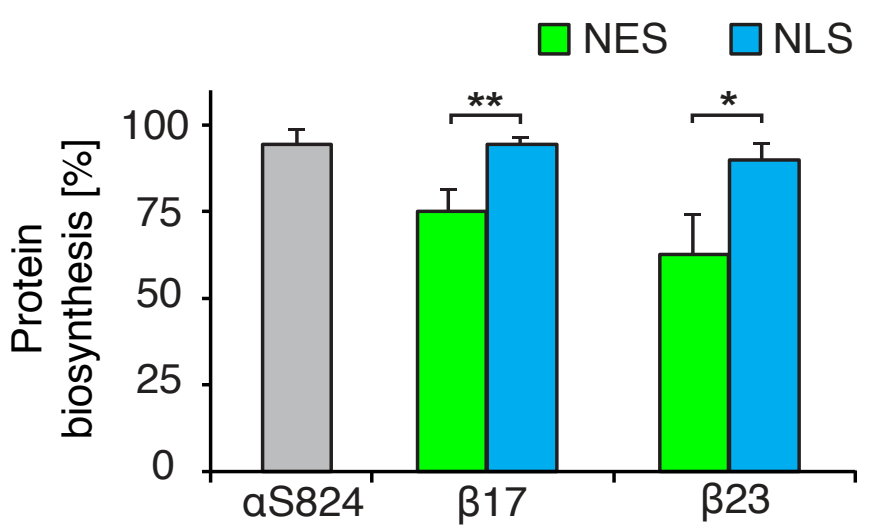

D

Wt

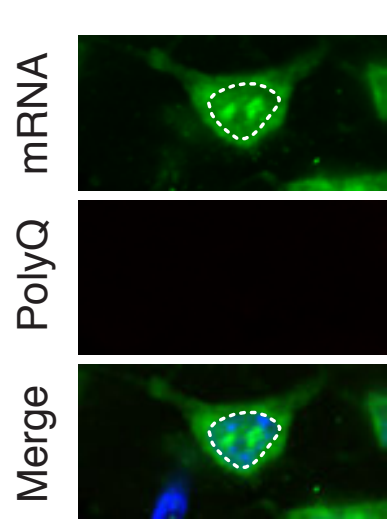

$\mathrm{R} 6 / 2$

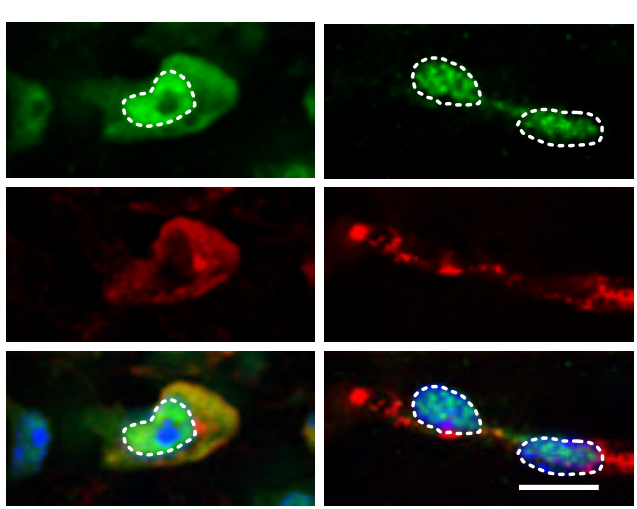

E

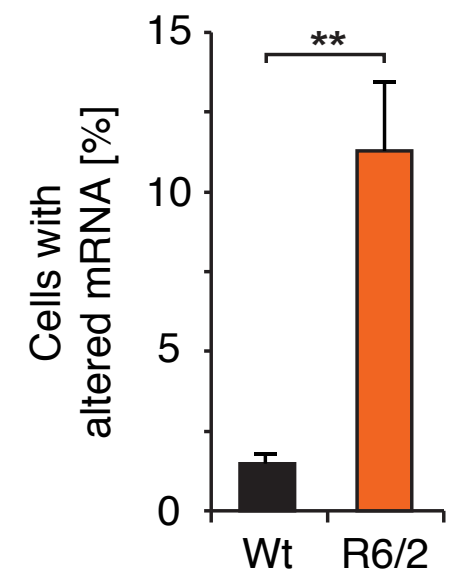


Woerner et al. Fig.4

A

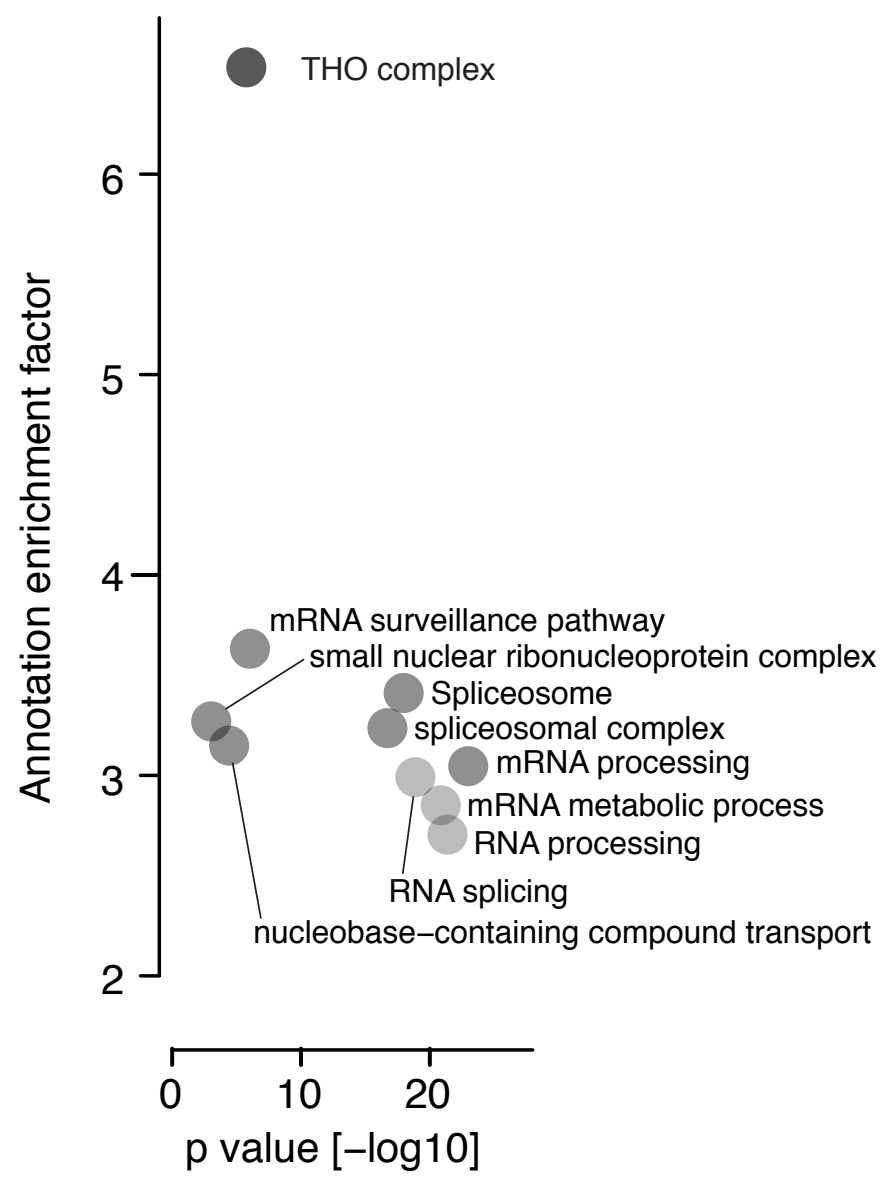

B

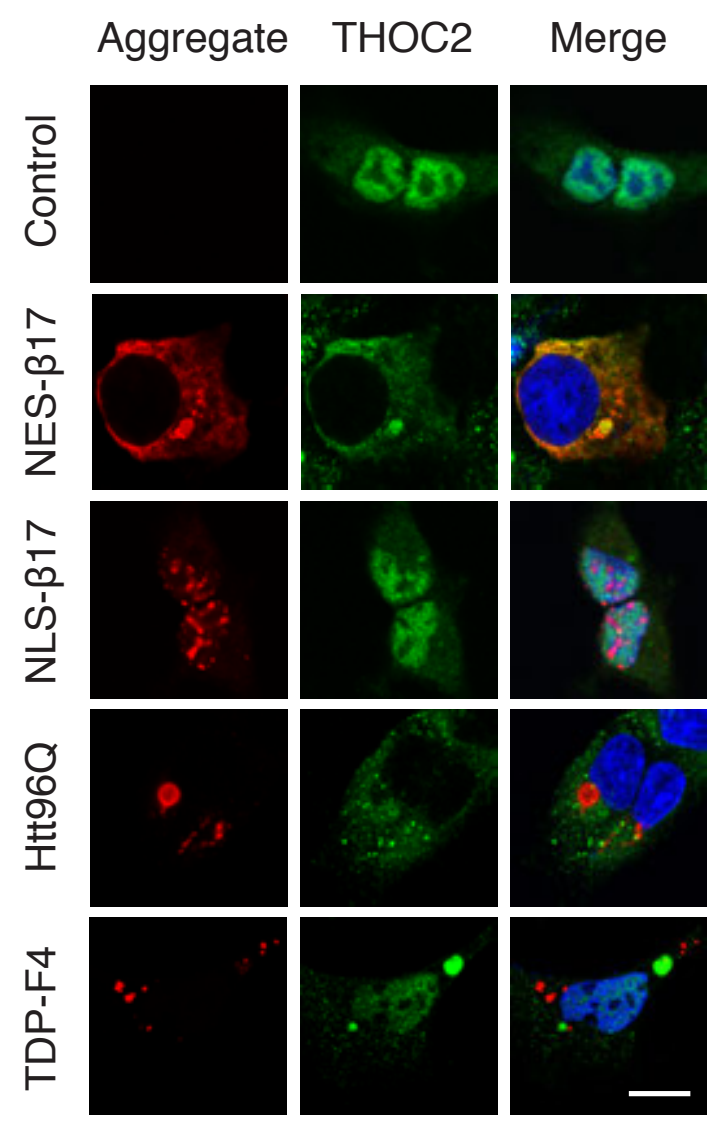




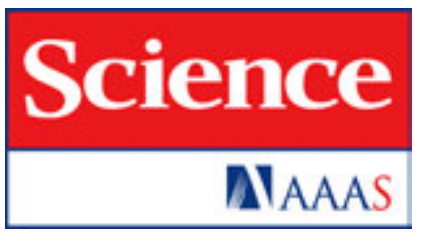

\section{Supplementary Materials for}

Cytoplasmic protein aggregates interfere with nucleo-cytoplasmic transport of protein and RNA

Andreas C. Woerner, Frédéric Frottin, Daniel Hornburg, Li R. Feng, Felix Meissner, Maria Patra, Jörg Tatzelt, Matthias Mann, Konstanze F. Winklhofer, F. Ulrich Hartl, and Mark S. Hipp

correspondence to: hipp@biochem.mpg.de; uhartl@biochem.mpg.de

\section{This PDF file includes:}

Materials and Methods

Figs. S1 to S11

Captions for Tables S1 to S3

Other Supplementary Materials for this manuscript includes the following:

Tables S1 to S3 


\section{Materials and Methods}

Cell Culture, Transfection and Immunoblotting

HEK293T and SH-SY5Y cells were cultured in Dulbecco's modified Eagle's medium (DMEM) (Biochrom KG), supplemented with $10 \%(\mathrm{v} / \mathrm{v})$ fetal bovine serum (FBS), $100 \mathrm{IU} / \mathrm{ml}$ penicillin, $100 \mu \mathrm{g} / \mathrm{ml}$ streptomycin sulfate, $2 \mathrm{mM} \mathrm{L}$-glutamine and nonessential amino acid cocktail (all from GIBCO). Transient transfections were performed by electroporation (GenePulser XCell System, Bio-Rad) or by lipofection with Lipofectamin 2000 (Invitrogen) according to the manufacturer's instruction.

Immunoblots were developed using Luminata Classico Western HRP substrate (Millipore), and analyzed using a LAS-3000 image reader (Fujifilm) and the AIDA software (Raytest).

Primary cortical neuron cultures. Cerebral cortices from embryonic day 17 (E17) C57BL/6J mouse embryos were dissected into ice-cold PBS and dissociated in $0.25 \%$ trypsin (Life Technologies) for $30 \mathrm{~min}$ at $37^{\circ} \mathrm{C}$. Following digestion with trypsin, cortical tissues were rinsed and further dissociated with pipette tips. The cell suspension was centrifuged at 1,000 rpm for $5 \mathrm{~min}$ and cell pellets were resuspended in Neurobasal medium (Life Technologies). Cortical neurons were plated in 24-well plates on coverslips coated with poly-L-lysine (BD BioCoat, BD Biosciences) and laminin ( $5 \mu \mathrm{g} / \mathrm{ml}$; Life Technologies) for FISH/immunocytochemistry analyses. Cells were plated in $10 \mathrm{~cm}$ plates coated with poly-L-lysine and laminin for proteomic studies. Neurons were cultured in Neurobasal medium supplemented with B27 (Life Technologies) and GlutaMAX (Life Technologies) in a 5\% $\mathrm{CO}_{2}$ incubator.

Primary cortical neuron transfection. DIV7 neurons (days in vitro 7) were transfected using the CalPhos Mammalian Transfection Kit (Clontech) according to manufacturer's instructions. Briefly, culture medium was removed and Opti-MEM (Life Technologies) was added to each well/plate prior to transfection. DNA-Ca ${ }^{2+}$-phosphate precipitate was prepared by adding a solution containing plasmid DNA and $\mathrm{CaCl}_{2}$ to $2 \mathrm{x}$ HBS. The DNA mixture was incubated at room temperature for $20 \mathrm{~min}$ and added drop wise to cultured cortical neurons. After incubating for $1 \mathrm{~h}$ the transfection mixture was replaced by the original culture medium. After 2-3 days of protein expression, cortical neurons were fixed in $4 \%$ paraformaldehyde $/ 4 \%$ sucrose in PBS for $10 \mathrm{~min}$, permeabilizied, hybridized and antibody stained as described for HEK/SH-SY5Y cells.

\section{Plasmids}

The plasmids expressing myc-tagged Htt96Q, $\alpha \mathrm{S} 824, \beta 17$ and $\beta 23$ were described previously $(9,31)$. NES- $\alpha \mathrm{S} 824 / \beta 17 / \beta 23$ were generated by integrating a consensus NES (LELLEDLTL) behind the Myc tag of $\alpha$ S824, $\beta 17$, and $\beta 23$. NLS- $\alpha S 824 / \beta 17 / \beta 23$ were generated by inserting 2 repeats of the SV40 derived NLS (DPKKKRKV) N-terminal to the Myc-tag of $\alpha$ S824, $\beta 17$, and $\beta 23$. NLS- $\beta 23(K 7 T, K 15 T$ ) was generated by site directed mutagenesis of NLS- $\beta 23$. For generating EGFP fusion proteins, $\alpha \mathrm{S} 824$ or the $\beta$ proteins were fused $\mathrm{N}$-terminal of EGFP (PCR amplified from $\mathrm{pEGFP-N1}$, Clontech), 
connected via a flexible 16 amino acid long linker sequence

(TSGSAASAAGAGEAAA;(32)).

S-GFP contains both an NES and an NLS and was generated by insertion of a fusion of EGFP (Clontech) with the described NES and NLS together with an HA-tag into the $\mathrm{KpnI}$ and XbaI sites of pcDNA3.1 myc-His A (Invitrogen). TDP-F4 was received from Addgene (TDP-43-EGFP construct 4, (19)).

\section{Antibodies}

The following primary antibodies were applied for immunofluorescence or immunoblotting: c-Myc (9E10), Santa Cruz (Sc-40); c-Myc-Cy3 (9E10), Sigma, (C6594); polyglutamine (3B5H10), Sigma, (P1874); NF-kB p65 (XP, D14E12), Cell Signaling Technology (\#8242); nuclear pore complex proteins (Mab414), Abcam (ab24609); GAPDH (6C5), Chemicon (MAB374); importin al (KpnA2), Abcam (ab70160); THOC2, Sigma (HPA047921); Neuro-Chrom Pan Neuronal MarkerAlexa488, Millipore, (ABN2300A4); Histone-H3, Abcam (ab1791); importin $\alpha 3$ (KpnA4), Abcam (ab84735); NPM-1 (B23), Invitrogen (32-5200).

Secondary antibodies used: Mouse IgG-Alexa488, Cell Signaling Technology (\#4408); Mouse IgG-Alexa555, Cell Signaling Technology (\#4409); Rabbit IgG-Atto488, Sigma (18772); Rabbit IgG-Alexa555, Cell Signaling Technology (\#4413); Rabbit IgGFITC, Sigma (F9887); Mouse IgG-Peroxidase, Sigma (A4416); Rabbit IgG-Peroxidase, Sigma (A9169).

\section{Cell viability by MTT assay}

Cell viability was analyzed by measuring the capacity of cells to reduce MTT (3(4,5-dimethylthiazol-2-yl)-2, 5-diphenyltetrazolium bromide) to formazan 3 days after transfection with the indicated constructs. The medium was removed and $0.5 \mathrm{~mL}$ of $0.5 \mathrm{mg} / \mathrm{mL}$ MTT dissolved in DMEM was added for $1 \mathrm{~h}$ at $37^{\circ} \mathrm{C}$. Cells were lysed and dissolved by the addition of $0.5 \mathrm{~mL}$ of $20 \%$ SDS (w/v) and 50\% DMF (v/v), and the absorbance at $570 \mathrm{~nm}$ was determined (KC4 software, Synergy-HT, BioTek) (33).

Solubility of cytoplasmic and nuclear $\beta$-proteins

$24 \mathrm{~h}$ after transfection with the indicated construct, $1 \times 10^{6}$ HEK293T cells were collected and resuspended in $200 \mu \mathrm{L}$ PBS (Gibco, pH 7.4) on ice and divided into 2 equal fractions. Cells were lysed by addition of an equal volume of $2 \mathrm{x}$ lysis buffer $(2 \%$ Triton $\mathrm{X}-100,750 \mathrm{mM} \mathrm{NaCl}, 1 \mathrm{mM} \mathrm{KH} \mathrm{PO}_{4}, 3 \mathrm{mM} \mathrm{Na} 2 \mathrm{HPO}_{4}$, Protease Inhibitor Cocktail (Roche) and Benzonase Nuclease (Novagen), pH 7.4). Samples were vortexed and incubated with agitation for $45 \mathrm{~min}$ at $4^{\circ} \mathrm{C}$, before centrifugation at $20,000 \mathrm{xg}$ for $10 \mathrm{~min}$. The supernatant was completely removed and the pellet was suspended in an equal volume of $1 \mathrm{x}$ lysis buffer. Total samples were processed without centrifugation. Immunoblotting against Histone-H3 served as control for nuclear lysis, and GAPDH as loading control.

Immunoprecipitation of $\beta$-proteins variants from HEK293T cells

Cells were harvested $24 \mathrm{~h}$ post-transfection and lysed with RIPA buffer supplemented with Protease Inhibitor Cocktail (Roche) and Benzonase Nuclease (Novagen). Samples were briefly sonicated and incubated for $30 \mathrm{~min}$ at $4^{\circ} \mathrm{C}$. After 
centrifugation $\left(2,000 \mathrm{~g} ; 4^{\circ} \mathrm{C} ; 10 \mathrm{~min}\right)$ and determination of protein concentration $1 \mathrm{mg}$ of lysate was incubated with constant agitation with $50 \mu 1 \mu \mathrm{MACS}$ magnetic anti-cMYC beads (Miltenyi Biotec). Samples were then added to pre-equilibrated columns, washed three times with lysis buffer, once with PBS and then eluted with loading dye. Eluates and lysate fractions were subsequently analyzed by SDS-PAGE and immunoblotting.

Immunofluorescence

HEK293T and SH-SY5Y cells were grown on poly-L-lysine coated coverslips (German glass, BD BioCoat, BD Biosciences) for 24-48 h. Cells were fixed in 4\% paraformaldehyde in PBS for 10 min, permeabilized with $0.1 \%(\mathrm{v} / \mathrm{v})$ Triton X-100 in PBS for $5 \mathrm{~min}$, and washed with PBS. Cells were stained with primary antibodies at a dilution of 1:200 to 1:1,000 in PBS containing 1\% BSA for $1 \mathrm{~h}$ at room temperature or overnight at $4^{\circ} \mathrm{C}$, washed with PBS, followed by fluorescent dye-conjugated secondary antibodies at a dilution of 1:1,000 for several hours at room temperature. Cells were incubated in PBS containing $0.25 \mu \mathrm{g} / \mathrm{mL}$ DAPI for $10 \mathrm{~min}$, washed in PBS and mounted in fluorescence mounting medium (DAKO).

To detect amyloid species, cells were additionally stained with 100 nM NIAD-4 (ICX Nomadics) for 30 min after fixation and permeabilization.

FRAP (fluorescence recovery after photo bleaching)

HEK293T cells were transiently transfected with vectors expressing C-terminally EGFP tagged $\beta$ proteins. After $24 \mathrm{~h}$, spherical regions of interest (ROI) were bleached for $\sim 1 \mathrm{~s}$ reaching $5-20 \%$ of original fluorescence intensity in the respective ROI, and recovery was followed over time $\left(\right.$ at $\left.37^{\circ} \mathrm{C}\right)$. Fluorescence intensities were normalized to $100 \%$ before and $0 \%$ after bleaching (first recording after bleaching).

Image acquisition

Confocal imaging was performed at the Imaging Facility of Max Planck Institute of Biochemistry, Martinsried, on a LEICA (Wetzlar, Germany) TCS SP2 AOBS, a LEICA TCS SP8 AOBS, or a ZEISS (Jena, Germany) LSM780 confocal laser scanning microscope.

In multi-fluorescent samples, single stained control samples were used to adapt emission bandwidth settings and signal amplification intensities to minimize spectral overlap, and to exclude erroneous co-localizations.

Fluorescence in situ hybridization (RNA FISH)

To detect polyA RNA in cells, HEK293T, SH-SY5Y cells or primary neurons were fixed in 4\% paraformaldehyde in saline-sodium citrate, $\mathrm{pH} 7.0$ (SSC, ultrapure, Sigma) for $10 \mathrm{~min}$, permeabilized with $0.1 \%(\mathrm{v} / \mathrm{v})$ Triton X-100 in SSC for $5 \mathrm{~min}$, and washed with FISH buffer (SSC, 10\% formamide) for $10 \mathrm{~min}$ at $37^{\circ} \mathrm{C}$. (d) $\mathrm{T}_{30}$-Cy5 oligonucleotide was applied at $0.1 \mu \mathrm{M}$ in FISH buffer including $10 \%$ dextran sulfate (average molecular weight $>500 \mathrm{kDa}$, Sigma) for $2 \mathrm{~h}$ in a humidified chamber at $37^{\circ} \mathrm{C}$. Cells were washed 2 times in FISH buffer at $37^{\circ} \mathrm{C}$. Antibody and DAPI staining followed as described. 
Fluorescence in situ hybridization on mouse brain tissue (RNA FISH)

All animal experiments were performed in compliance with institutional and governmental regulations.

R6/2 mice and wild-type litter mates were received from The Jackson Laboratory (B6CBA- $\operatorname{Tg}(\mathrm{HDexon} 1) 62 \mathrm{Gpb} / 1 \mathrm{~J})$. Mice were anaesthetized with $5 \%$ chloral hydrate in PBS and transcardially perfused with $4 \%$ paraformaldehyde/PBS. The brains were postfixed for $2 \mathrm{~h}$ in $4 \%$ paraformaldehyde $/ \mathrm{PBS}$ at $4^{\circ} \mathrm{C}$ and cryoprotected in $30 \%$ sucrose $(\mathrm{w} / \mathrm{v}) / \mathrm{PBS}$ for $24 \mathrm{~h}$ at $4^{\circ} \mathrm{C}$. Brains were embedded in Tissue-Tek O.C.T. Compound (Sakura) at $-20^{\circ} \mathrm{C}, 20 \mu \mathrm{m}$ coronal sections were cut on a freezing microtome (Leica), dried on glass slides overnight on air and kept at $-20^{\circ} \mathrm{C}$ until use.

Sections were thawed and washed in prewarmed FISH tissue buffer (SSC, $10 \%(\mathrm{v} / \mathrm{v})$ formamide, $0.3 \%$ Triton X-100 (v/v)) at $45^{\circ} \mathrm{C}$. Hybridization to (d) $\mathrm{T}_{30}$-Cy5 oligonucleotides was performed in a humidified chamber in prewarmed FISH tissue buffer including $10 \%$ dextran sulfate (average molecular weight $>500 \mathrm{kDa}$, Sigma) for 1$3 \mathrm{~h}$ at $43^{\circ} \mathrm{C}$. Afterwards, sections were washed with FISH tissue buffer for $10 \mathrm{~min}$ at $45^{\circ} \mathrm{C}$.

For immunostaining, the hybridized sections were washed in $0.3 \%$ TritonX-100 in PBS, before overnight treatment with the indicated antibodies. Sections were subsequently incubated in $0.25 \mu \mathrm{g} / \mathrm{mL}$ DAPI in PBS for $10 \mathrm{~min}$ and washed 2 times in PBS before covering them with a glass slide (Menzel Gläser \#1, Thermo Scientific) in fluorescence mounting medium (DAKO).

For immunostaining of THOC2 equal sections from the brains of mice used for RNA hybridization analysis were thawed and washed in $0.3 \%$ TritonX-100/PBS.

Sections were stained with THOC2 (HPA047921, Sigma) and polyglutamine (3B5H10, Sigma) antibodies in $1 \%$ BSA, $0.3 \%$ TritonX-100/PBS overnight at $4^{\circ} \mathrm{C}$, followed by fluorescently labeled secondary antibodies and DAPI staining. Sections were washed with PBS and mounted in fluorescence mounting medium (DAKO).

Examining nuclear protein import/export with S-GFP

$3 \times 10^{6}$ HEK293T cells in 400 $\mu$ L DMEM supplemented with 25\% FBS were transfected by electroporation with $3 \mu \mathrm{g}$ S-GFP in addition to $25 \mu \mathrm{g}$ of plasmids encoding the $\beta$ proteins, Htt96Q, TDP-F4 or empty pcDNA3.1. After $40 \mathrm{~h}$ cells were treated with either $10 \mathrm{ng} / \mathrm{mL}$ CMR1 inhibitor Leptomycin B (LMB, Sigma) or DMSO for $15 \mathrm{~min}$ before fixation and immunostaining of aggregates.

The relative concentration of S-GFP in cytoplasm and nucleus was quantified by measuring the fluorescence intensity ratio in 75-150 individual cells per sample and condition in 3 independent repeats. The fluorescence intensities in cytoplasm and nucleus were determined using ImageJ (Rasband, W.S., ImageJ, U. S. National Institutes of Health, Bethesda, Maryland, USA, http://imagej.nih.gov/ij/, 1997-2012.).

NF- $\mathrm{KB}$ activation and translocation

HEK293T cells were transiently transfected with vectors expressing $\beta$ proteins, Htt96Q, TDP-F4, or with empty pcDNA3.1. After $40 \mathrm{~h}$, cells were treated for an additional 30 min with $20 \mathrm{ng} / \mathrm{mL}$ recombinant human TNF $\alpha$ (Biomol $\mathrm{GmbH}$ ), where 
indicated. At least 100 cells for each condition were evaluated in each experiment for assessment of NF- $\mathrm{kB}$ translocation by fluorescence microscopy.

Single cell quantification of total polyA RNA

HEK293T cells were fixed $24 \mathrm{~h}$ after transfection, permeabilized, and stained for polyA RNA, aggregating proteins, and nuclear DNA, as described above. The relative total polyA RNA fluorescence intensities of single cells containing aggregates was quantified (ImageJ, integrated signal intensity over the complete area of single cells) and normalized to untransfected cells on the same focal area ( $\geq 3$ independent experiments, $n$ $=4-13$ cells, each).

Absolute quantification of cytoplasmic mRNA

HEK293T cells were transfected with the indicated construct. After $40 \mathrm{~h}$, cells were harvested and one aliquot of each sample was set aside to control for equal protein content by immunoblotting against GAPDH. mRNA purification was performed with polyA+ RNA polystyrene-latex particles of uniform size ( $1 \mu \mathrm{m}$ diameter) covalently linked to $\mathrm{dC}_{10} \mathrm{~T}_{30}$ oligonucleotides (Oligotex, Qiagen), according to the manufacturers' instructions with the modification that no spin columns were used during the elution to facilitate quantification. The supernatant containing the purified cytoplasmic mRNA was immediately transferred to a separate tube for photometric determination of concentration and purity (absorption at 260/280 nm, NanoDrop 1000 spectrophotometer, ThermoScientific).

Actinomycin $\mathrm{D}$ was applied for general transcriptional inhibition by RNA Polymerase II at $5 \mu \mathrm{g} / \mathrm{mL}$ for $16 \mathrm{~h}$ (34) to cells transfected with an empty control plasmid.

THOC2 down-regulation by siRNA

Cells were transfected with either THOC2 siRNA (Thermo Scientific, "SMARTpool ON-TARGET plus" human THOC2 siRNA L-025006-01) or by non-targeting control siRNA targeting firefly luciferase mRNA (Thermo Scientific, "SMARTpool ONTARGET plus" non-targeting siRNA \#2, D-001810-02-05) using DharmaFECT1 (Thermo Scientific) transfection reagent as recommended by the manufacturer. Experiments were performed $72 \mathrm{~h}$ after siRNA transfection. The cellular knockdown of THOC2 2 was verified by immunoblotting and was $>85 \%$ efficient.

${ }^{35} \mathrm{~S}-$ Met radioactive pulse labeling

HEK293T cells were transfected with $\beta$ proteins and control plasmids. After $24 \mathrm{~h}$ the medium was switched to prewarmed cysteine-/methionine-free DMEM (GIBCO) without FBS. After $20 \mathrm{~min}, 50 \mu \mathrm{Ci} / \mathrm{mL}^{35} \mathrm{~S}-\mathrm{Met}$ (Perkin Elmer) was added and cells were incubated for another $20 \mathrm{~min}$ at $37^{\circ} \mathrm{C}$. Cells were washed 3 times in ice cold PBS and lysed for $30 \mathrm{~min}$ in 1\% Triton X-100 in PBS including Complete protease inhibitor cocktail (Roche) and Benzonase (Novagen). Samples were analyzed on a NuPAGE $4-12 \%$ gradient BisTris SDS Mini Gel (Invitrogen). The gel was stained with Serva Blue G (Serva), dried on a filter paper (Whatman), and analyzed by fluorography (FLA-2000, Fujifilm; Aida Image Analyzer). 


\section{Proteomics}

Sample Preparation. Cortical neurons were lysed in $1 \mathrm{ml}$ lysis buffer (1\% NP-40, 1

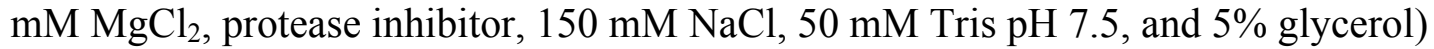
with addition of $5 \mu \mathrm{l}$ benzonase. After $30 \mathrm{~min}$, cell lysates were collected and centrifuged at $4,000 \mathrm{x} \mathrm{g}$ for $15 \mathrm{~min}$ at $4^{\circ} \mathrm{C}$. Cell lysates were incubated at room temperature for 15 min in the presence of $50 \mu 1 \mu \mathrm{MACS}$ Anti-GFP beads (Miltenyi Biotec) and added to pre-equilibrated columns. Subsequently, the columns were washed 4 times with Wash Buffer I (0.05\% NP-40, $150 \mathrm{mM} \mathrm{NaCl}, 50 \mathrm{mM}$ Tris $\mathrm{pH} 7.5,5 \%$ glycerol) and twice with Wash Buffer II (150 mM NaCl, $50 \mathrm{mM}$ Tris $\mathrm{pH} 7.5,5 \%$ glycerol). After incubating with $25 \mu 1$ Elution Buffer I (8M Urea, 1mM DTT, in 50mM Tris pH 7.5) containing LysC and 60 min with $25 \mu 1$ Elution buffer II (50mM, $5 \mathrm{mM}$ chloroacetamide, Tris $\mathrm{pH} 7.5$ ) containing trypsin, samples were eluted with $50 \mu$ l Elution Buffer II. To the eluate 1/10 volume thiourea /urea $(2 \mathrm{M} / 6 \mathrm{M})$ were added and the samples were digested overnight in the presence of trypsin before proteomic analysis.

$L C-M S / M S$. Before the peptide mix was subjected to LC-MS, salts were removed via C18 StageTips (35). We used liquid chromatography (Thermo Scientific EASY-nLC 1000 HPLC) with in- house packed columns (75 $\mu \mathrm{m}$ inner diameter, $20 \mathrm{~cm}$ length, 1.9 $\mu \mathrm{m} \mathrm{C} 18$ particles (Dr. Maisch $\mathrm{GmbH}$, Germany)) to separate peptides at $50^{\circ} \mathrm{C}$ in a 104 min gradient from $98 \%$ buffer A $(0.5 \%$ formic acid ) and $2 \%$ buffer B $(0.5 \%$ formic acid to $80 \%$ acetonitrile) to $60 \%$ buffer B at $400 \mathrm{nl} / \mathrm{min}$. An Orbitrap mass spectrometer (Orbitrap elite (36), Thermo Fisher Scientific) was directly coupled to the liquid chromatography column via a nano electrospray source. The mass spectrometer was operated in a data dependent mode. The survey scan range was set to 300 to $1.650 \mathrm{~m} / \mathrm{z}$, with a resolution of 120,000 . Up to the 5 most abundant isotope patterns with a charge $\geq$ 2 were subjected to collision induced dissociation at a normalized collision energy of 35 , an isolation window of $2 \mathrm{Th}$. Data were acquired using the Xcalibur software (Thermo Scientific).

Data analysis and statistics. We employed the MaxQuant software (v 1.5.02.22) (37) and Andromeda search engine (38) to process MS raw data searching against the UniProtKB mouse FASTA database (06/2012) using settings as described before (39). The retention time window to match MS/MS features to MS features across the samples was set to $0.7 \mathrm{~min}$. Potential contaminants $(\mathrm{n}=247)$ and proteins only identified with site modifications on the peptide level were strictly excluded from the analysis. GFP was confidently detected with up to 12 unique peptides at similar abundance in the individual controls and $\beta 17-$ GFP IPs (see Table S3A, CON_Q9U6Y5). Quantification and normalization was performed using the MaxLFQ label free algorithms (40). For bioinformatic analysis and visualization we used PERSEUS, which is part of MaxQuant and the $\mathrm{R}$ framework (41). Missing values were imputed with a normal distribution (width $=0.3$; shift $=1.8$ ). We performed a Fisher-exact test for annotations on t-test significant (unequal variances, permutation-based false discovery rate of $5 \%$ and S0 of 1 (42)) proteins enriched for $\beta 17$ (150 proteins) against the background ( 979 proteins). We filtered for categories occurring in the $\beta 17$ interactome more than 6 times and applied a Benjamini Hochberg false discovery rate cut-off at $2 \%$. To visualize highly enriched annotations (GOBP, GOCC, GOMF, KEGG, Uniprot Keywords), we plotted resulting pvalues against the enrichment factor. THOC4 is not annotated as a THO complex 
member in GOCC. To have a correct estimation of the enrichment, we manually supplemented GOCC annotation "Tho complex" with THOC4. To estimate the amount of low complexity regions we employed webpage integrated pSEG algorithms (http://fasta.bioch.virginia.edu/fasta www2/fasta www.cgi? rm=misc1\&pgm=seg, Copyright (C) 1988, 2006 by William R. Pearson and the University of Virginia; and (43)). To estimate the amount of disordered regions and the longest disordered stretch per protein we accessed the $\mathrm{D} 2 \mathrm{P} 2$ database $(44)$ with the 'rjson' package in $\mathrm{R}(41,45)$ and retrieved the respective IUPred-L (46) information via Uniprot identifier. We performed a Mann-Whitney test on the retrieved data for $\beta 17$ interactors compared to the retrieved data for all quantified proteins to test for significant differences between both populations. To visualize the distribution we plotted box plots (47) with whiskers indicating the $5 \%$ and $95 \%$ quantile, the box depicting the $25 \%$ and $75 \%$ quantile and the median (horizontal dashed line).

\section{Statistics}

Unpaired Student's $\mathrm{t}$ test was used to determine significant differences between samples (significance levels: ${ }^{*}$ ) for $\mathrm{p}<0.05,(* *)$ for $\mathrm{p}<0.01,(* * *)$ for $\mathrm{p}<0.001$ ). 


\section{NLS Myc $\beta>\beta>\beta>\beta>\beta>\beta$}

\section{Myc NES $\beta>\beta>\beta>\beta>\beta>\beta$}

B

ß17

'M DYEIKFH GD

GD NFDLNLD DS

GG DLQLQIR GP

GG RVHVHIH SS

SG KVDFHVN ND

GG DVEVKMH ${ }^{63}$

\section{$\beta 23$}

'M DYNIQFH NN

GN EIQFEID DS

GG DIEIEIR GP

GG RVHIQLN DG

HG HIKVDFH ND

GG ELQIDMH ${ }^{63}$

$\beta 17$

ATGGATTATGAGATCAAGTTCCACGGTGAT GGTGATAACTTCGACCTCAACCTCGACGATTCT GGTGGTGATCTTCAACTTCAGATCCGCGGCCCG GGTGGCCGTGTCCACGTCCACATCCACAGTAGT AGTGGTAAGGTCGACTTCCACGTCAACAACGAC GGCGGCGATGTTGAAGTTAAAATGCACTAG

\section{$\beta 23$}

ATGGATTATAACATCCAGTTCCACAATAAT GGTAATGAGATCCAGTTCGAGATCGACGATTCT GGTGGTGATATTGAAATTGAGATCCGCGGCCCG GGTGGCCGTGTCCACATCCAGCTCAACGATGGT CATGGTCACATCAAGGTCGACTTCCACAACGAC GGCGGCGAACTTCAAATTGATATGCACTAG

Fig. S1.

Aggregation-prone $\beta$-proteins do not contain amino acid or nucleotide repeats. (A) Schematic representation of aggregation-prone $\beta$-proteins designed to form six-stranded $\beta$-sheets targeted to the cytoplasm or nucleus. N-terminal c-Myc tag (red); SV40 derived nuclear localization sequence (NLS) (blue); optimized nuclear export sequence (NES) (green). (B) Amino acid sequences of $\beta 17$ and $\beta 23$. Polar and nonpolar amino acids are indicated in grey and yellow, respectively. (C) DNA sequence of $\beta 17$ and $\beta 23$. 
A

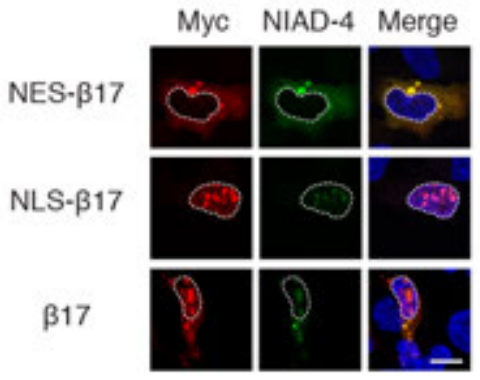

B

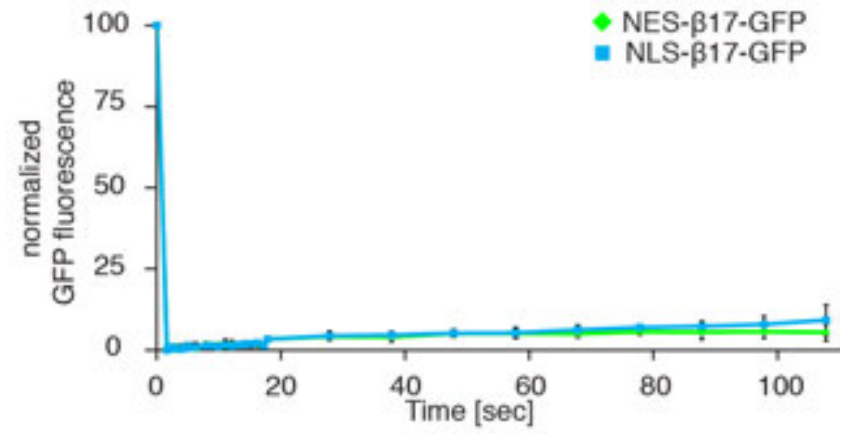

Fig. S2

Cytoplasmic and nuclear $\beta$-proteins inclusions are immobile and differ in binding to the amyloid-detecting dye NIAD-4. (A) NIAD-4 staining (green) of cells expressing $\beta 17$ proteins $24 \mathrm{~h}$ after transfection. Anti-Myc labelling (red) and nuclear DNA staining (blue). Dashed lines indicate the nucleus. Scale bar, $10 \mu \mathrm{m}$. (B) NES- $\beta 17-E G F P$ and NLS- $\beta 17-E G F P$ were expressed in HEK293T cells for $24 \mathrm{~h}$. Spots in cytoplasmic and nuclear inclusions were bleached for $\sim 1 \mathrm{~s}$ and fluorescence recovery was followed in 3-6 inclusions per condition per repeat over time. Data are represented as mean $+/-\mathrm{SD}, \mathrm{N}=3$. 


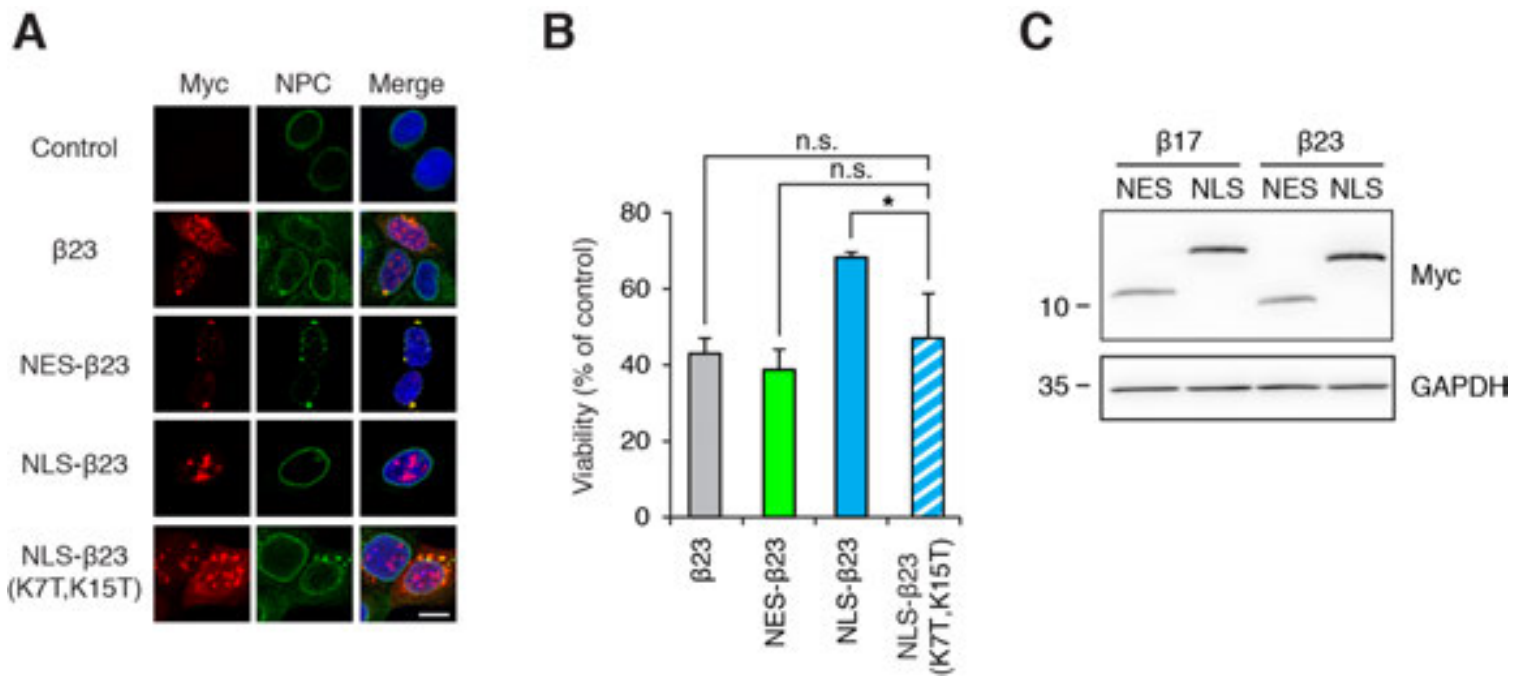

Fig. S3

Reduced toxicity of aggregation-prone proteins targeted to the nucleus. (A) Mutation of two lysine residues in the NLS prevented nuclear targeting. HEK293T cells were transfected with empty vector (Control) or different variants of $\beta 23$, and $24 \mathrm{~h}$ after transfection labelled with anti-Myc antibodies (red), anti-NPC antibodies (green) and nuclear DAPI staining (blue). Scale bar, $10 \mu \mathrm{m}$. (B) Viability of HEK293T cells expressing different variants of $\beta 23$ as measured by MTT assay $72 \mathrm{~h}$ after transfection. Values for proteins targeted to the cytoplasm (NES) or the nucleus (NLS) from experiments performed in parallel (Fig. 1D) are indicated for comparison. Data are mean $+\mathrm{SD}, \mathrm{N}=3$. n.s. non significant; *, $\mathrm{p} \leq 0.05$ from unpaired Student's $t$-test. (C) Total protein levels of $\beta$-proteins directed to the cytoplasm and nucleus. Cells were transfected with the indicated $\beta$-proteins and grown for $24 \mathrm{~h}$, followed by immunoblotting with antiMyc antibodies. 


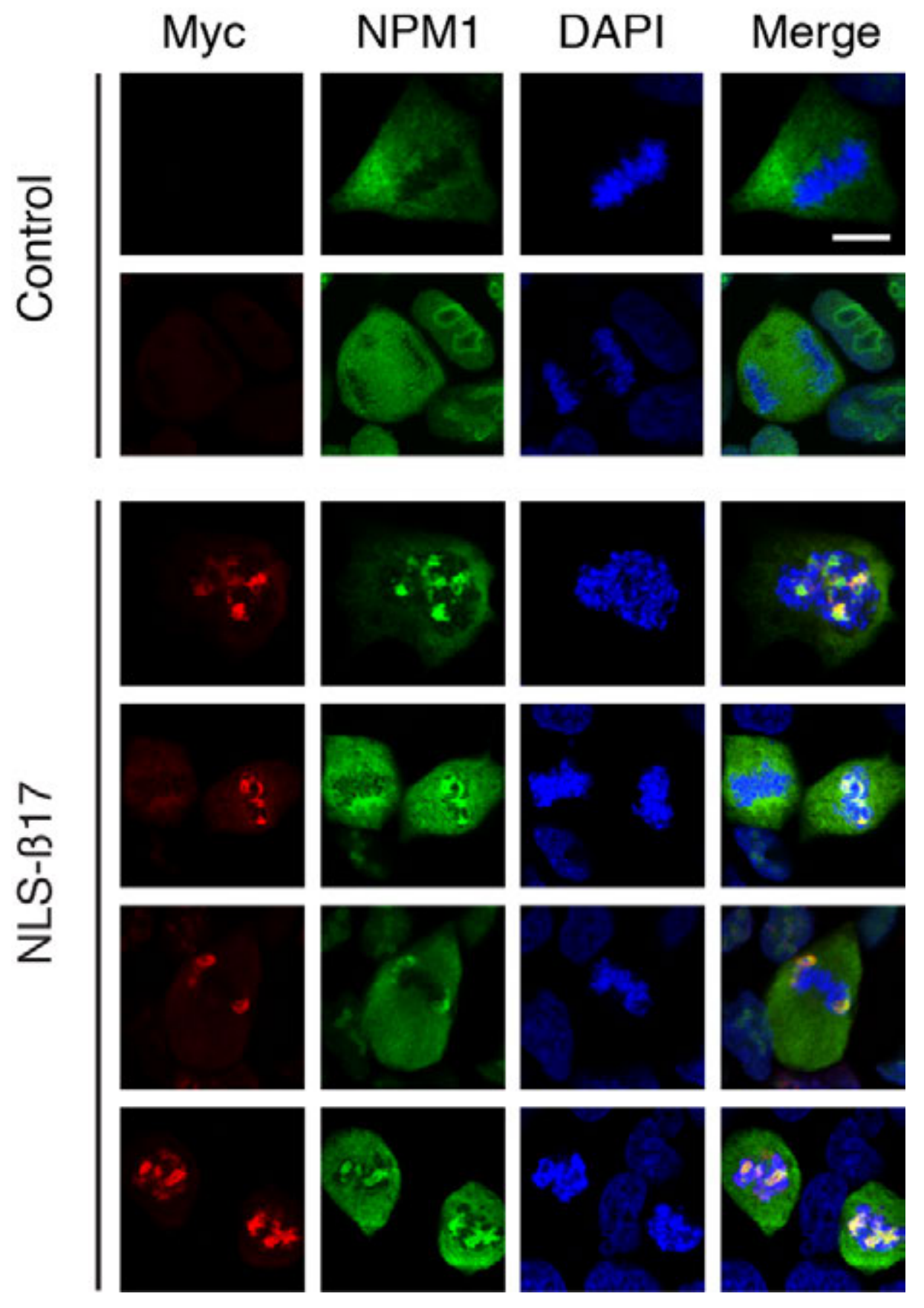

Fig. 54

Nuclear aggregates associate with NPM1 even when nucleoli are disassembled during mitosis. HEK293T were transfected with empty vector (Control) or NLS- $\beta 17$. $24 \mathrm{~h}-48 \mathrm{~h}$ after transfection cells were fixed and stained as described in the methods section. Endogenous NPM1 was detected using a monoclonal antibody (green), NLS$\beta 17$ using anti-Myc antibody (red) and DNA with DAPI (blue). Images of dividing cells at different stages of mitosis are shown. Scale bar, $10 \mu \mathrm{m}$. 


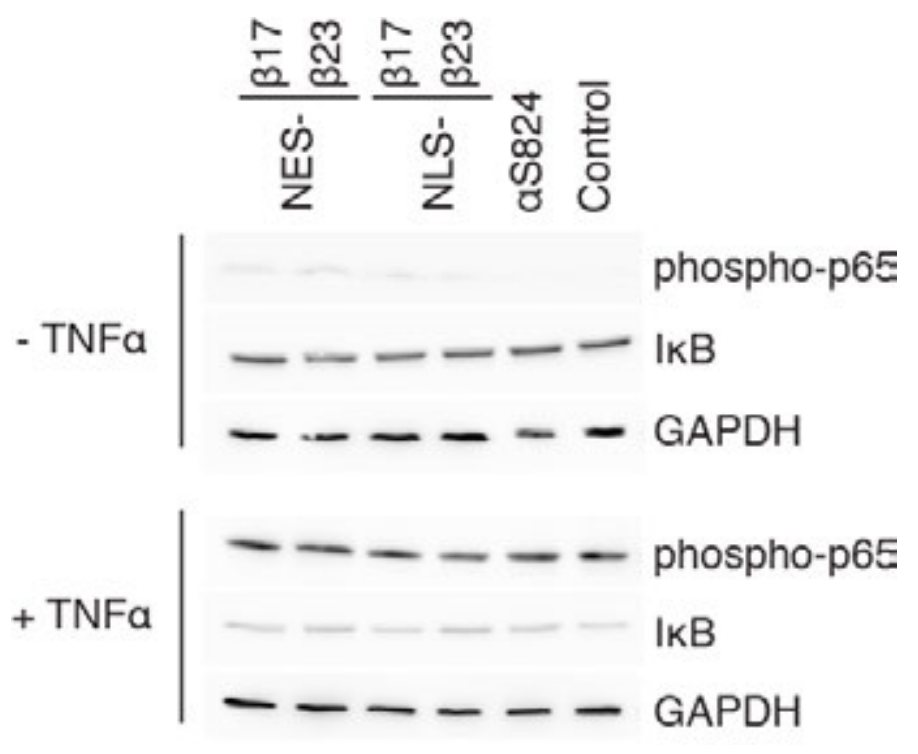

Fig. 55

Expression of $\beta$-sheet proteins does not alter degradation of IKB and phosphorylation of p65. HEK293T cells were transfected with the indicated constructs and grown for $40 \mathrm{~h}$, followed by incubation with or without TNF $\alpha$ for $15 \mathrm{~min}$ and

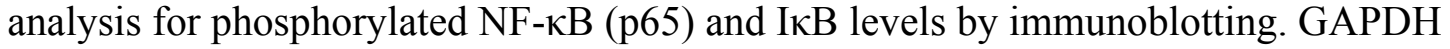
was analyzed as loading control. 
A

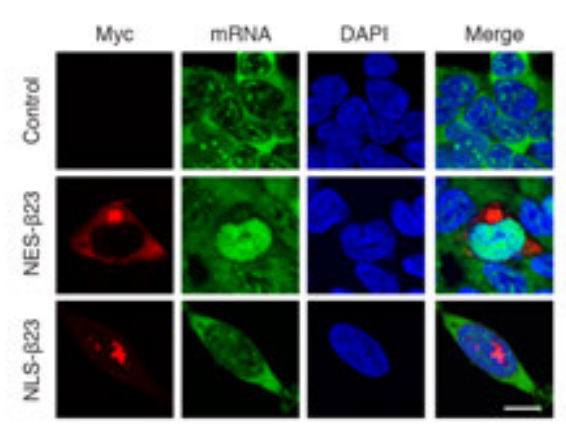

B

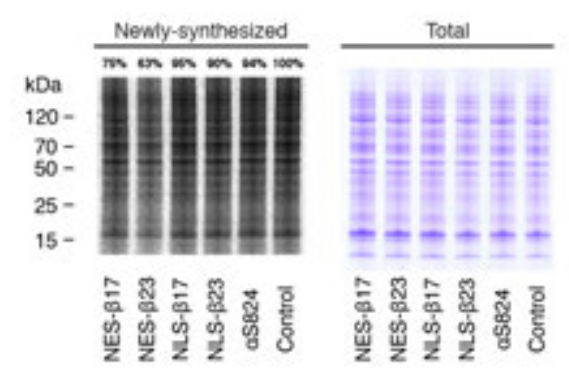

C

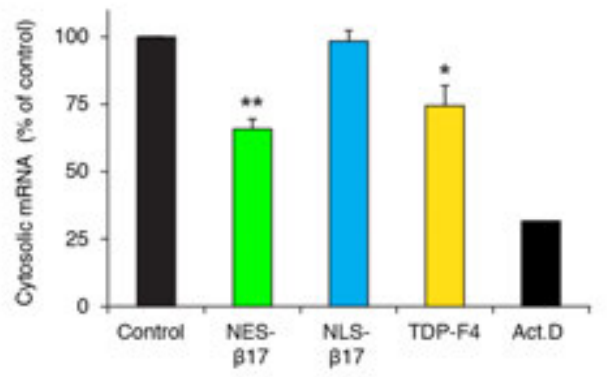

D

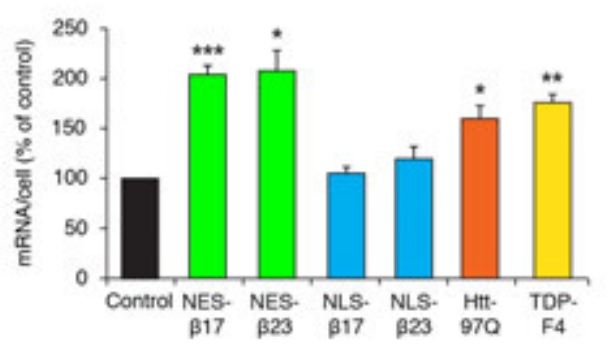

Fig. S6

Cytoplasmic aggregates inhibit nuclear RNA export and translation. (A) HEK293T cells were transfected with empty vector (Control), NES- $\beta 23$ or NLS- $\beta 23$. After $24 \mathrm{~h}$, cells were analyzed for total polyA RNA (poly(d)T30-Cy5, green) as in Fig. 3 and for $\beta$ proteins with anti-Myc antibodies (red). (B) Impairment of protein translation in cells expressing cytoplasmic $\beta$-proteins. $24 \mathrm{~h}$ after transfection with empty vector (Control) or the indicated constructs, HEK293T cells were pulse-labelled with ${ }^{35}$ S-Met for $20 \mathrm{~min}$. Cell lysates were analyzed by SDS-PAGE and fluorography to detect newly synthesized proteins (left). Total protein was stained with Coomassie blue (right). (C) Quantification of cytoplasmic mRNA isolated with Oligotex beads covalently linked to (d) $\mathrm{T}_{30}$ oligonucleotides. HEK293T cells were transfected with empty vector (Control), NES$\beta 17$, NLS- $\beta 17$ or TDP-F4, or were treated with the transcriptional inhibitor Actinomycin D (Act.D) for $16 \mathrm{~h}$. Data are mean $+\mathrm{SD}, \mathrm{N}=3$, except for Act.D treatment where the average of only 2 experiments is shown. *, $\mathrm{p} \leq 0.05,{ }^{* *}, \mathrm{p} \leq 0.01$. (D) Cytoplasmic aggregates are associated with a significant increase of total polyA RNA. Total polyA RNA fluorescence of single SH-SY5Y cells expressing cytoplasmic and nuclear $\beta$-proteins, Htt96Q, or TDP-F4 for $24 \mathrm{~h}$ relative to empty vector-transfected control cells. Data are mean $+\mathrm{SD}, \mathrm{N}=3 ; 4-15$ transfected cells were analyzed per experiment. *, $\mathrm{p} \leq 0.05,{ }^{* *}, \mathrm{p} \leq 0.01,{ }^{* *}, \mathrm{p} \leq 0.001$ using unpaired Student's $t$-test. 


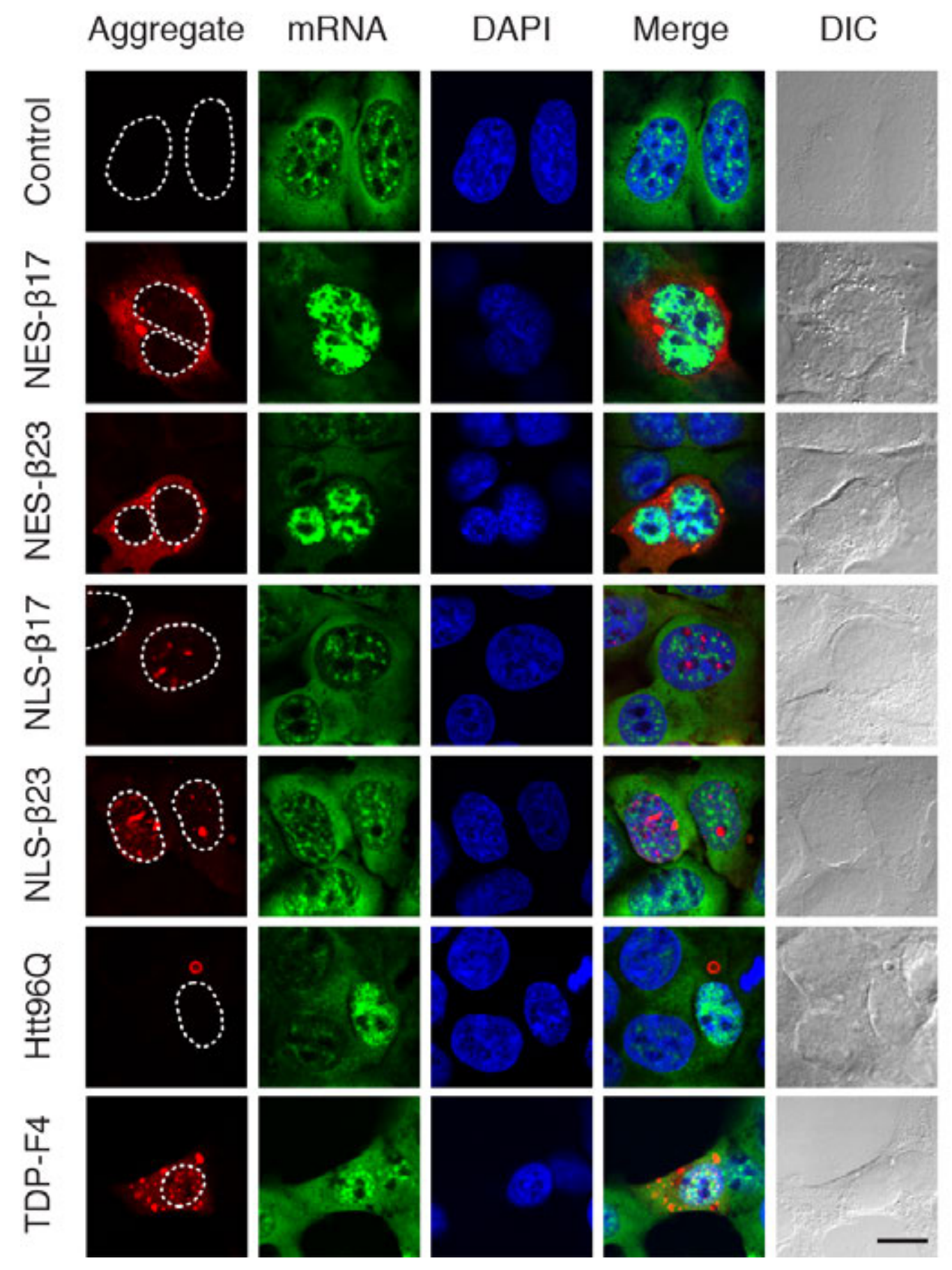

Fig. S7

Cytoplasmic aggregation inhibits export of mRNA in SH-SY5Y neuroblastoma cells. SH-SY5Y cells were transfected with empty vector (Control) or the indicated constructs (red). After $24 \mathrm{~h}$ cells were labelled for total polyA RNA (green) and nuclear DNA (blue). Differential interference contrast (DIC) images are also shown. Scale bar, $10 \mu \mathrm{m}$. 


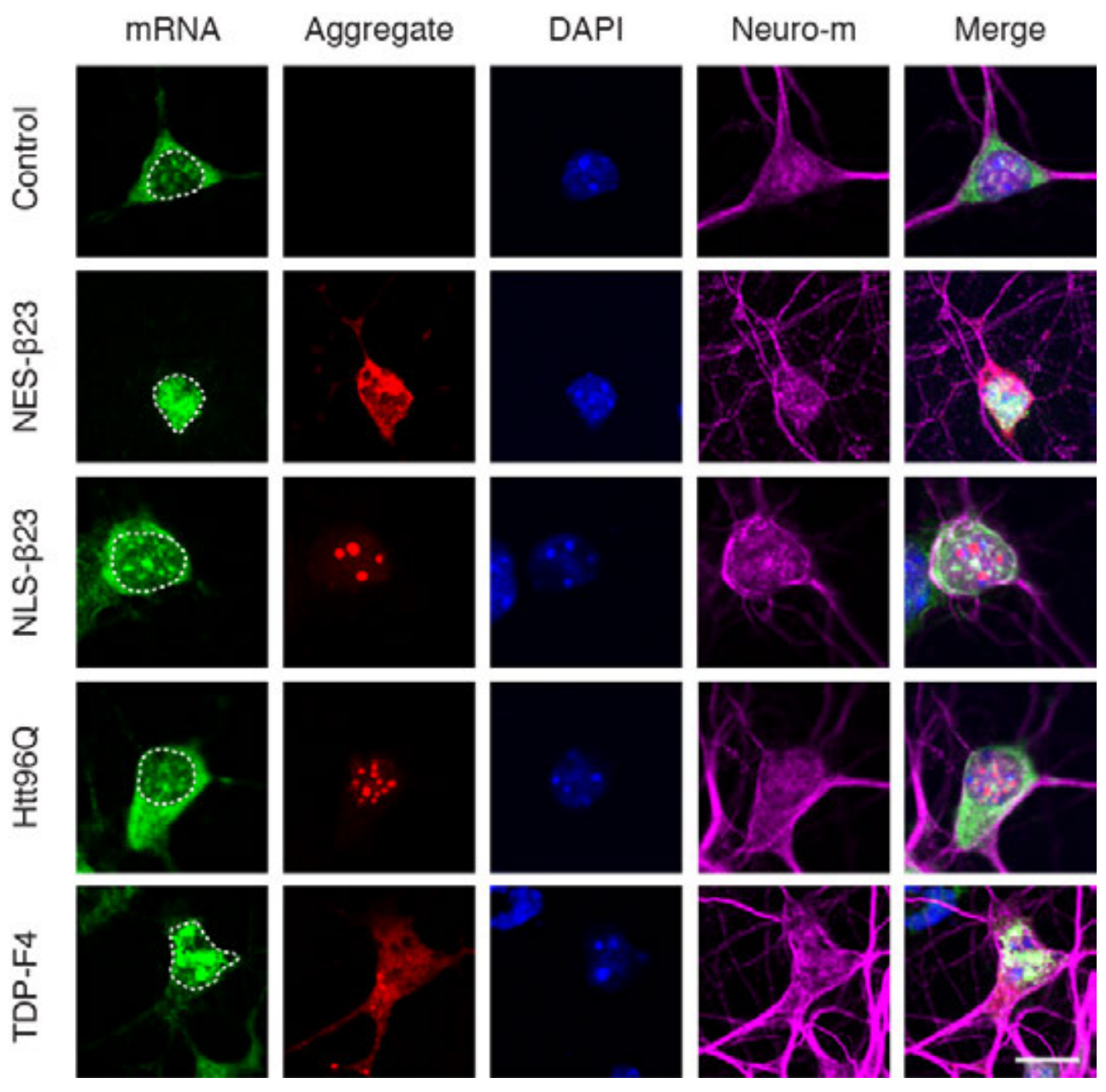

Fig. S8

Cytoplasmic aggregates cause nuclear mRNA retention in cortical mouse neurons. Embryonic cortical mouse neurons transfected with empty vector (Control), NES- $\beta 23$, NLS- $\beta 23$, Htt96Q, or TDP-F4 were labelled for mRNA (green), protein aggregates (red), nuclear DNA (blue) and with a monoclonal antibody cocktail against neuronal proteins (Neuro-m, magenta). Scale bar, $10 \mu \mathrm{m}$. 
A
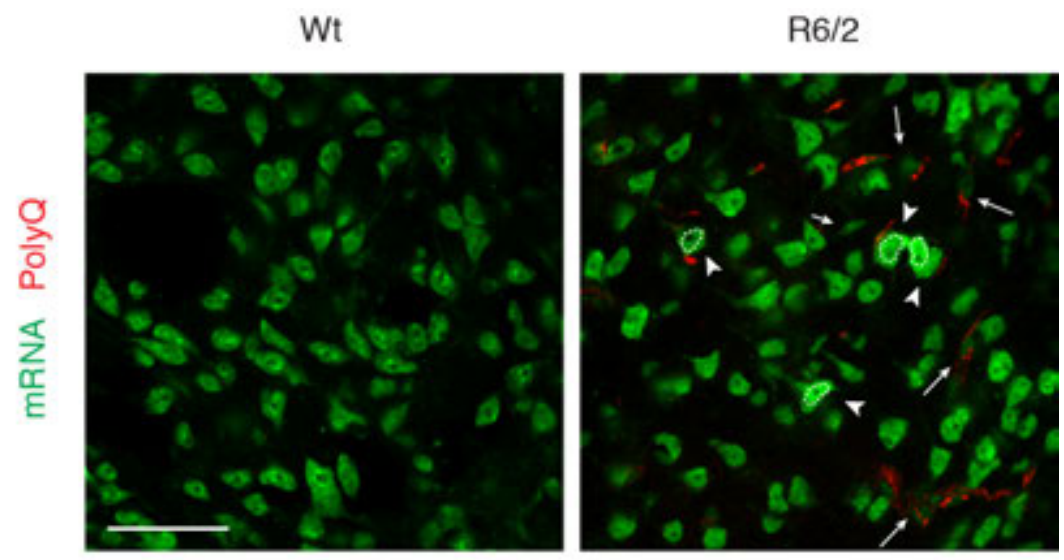

B
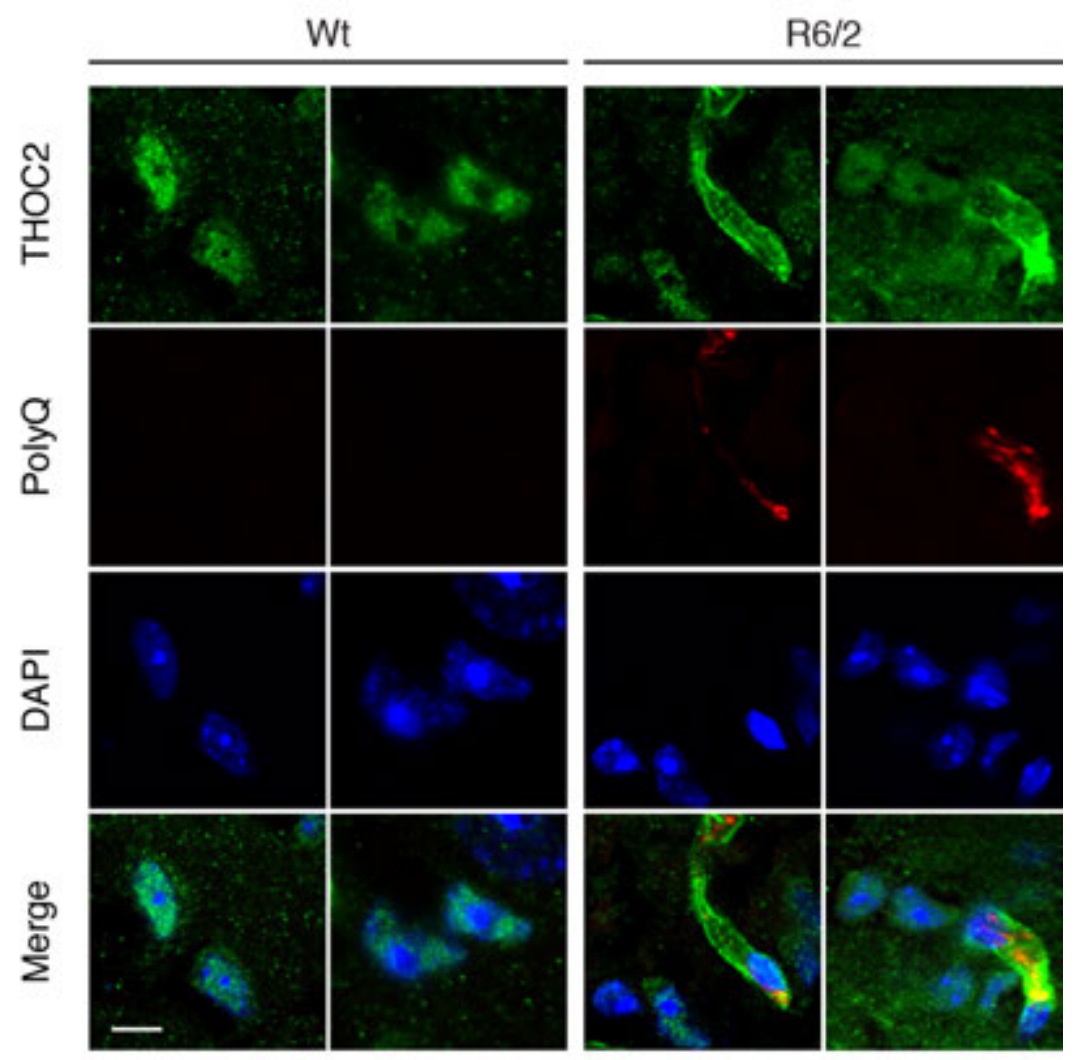

Fig. S9

mRNA and THOC2 distribution is altered in HD mouse brain. (A) Brain sections of 9-week-old wild-type (Wt) and R6/2 mice were stained for mRNA (green) and with an antibody specific for polyQ-expanded Htt (red). Neurons with nuclear accumulation of mRNA (arrowheads) and reduced mRNA levels (arrows) are indicated. Dashed lines indicate the nuclei of neurons with nuclear accumulation of mRNA. Scale bar, $50 \mu \mathrm{m}$. (B) Brain sections of 9-week-old wild-type (Wt) and R6/2 mice were stained for THOC2 (green), polyQ-expanded Htt (red), and nuclear DNA (blue). Scale bar, $10 \mu \mathrm{m}$. 


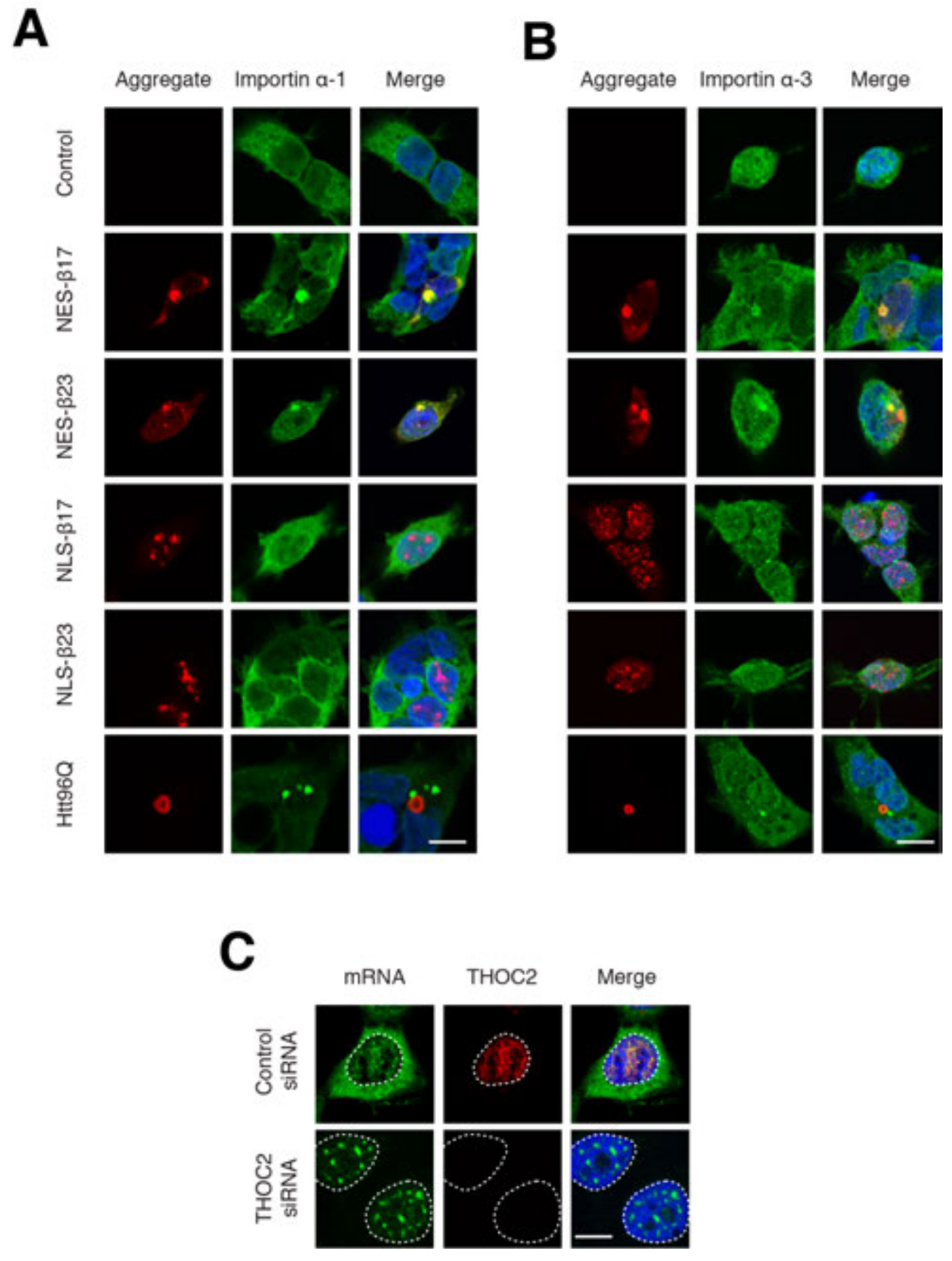

Fig. S10

Defective nuclear transport as a result of dislocated nuclear transport factors. (A) Immunofluorescence of HEK293T cells $24 \mathrm{~h}$ after transfection of empty vector (Control) or the indicated constructs, labelled with anti-Myc (red) and (A) anti-importin $\alpha-1$ (KPNA2), or (B) anti-importin $\alpha-3$ (green). Nuclear DNA was stained with DAPI (blue). Scale bars, $10 \mu \mathrm{m}$. (C) HEK293T cells treated with control siRNA or siRNA against THOC2 for 3 days were analyzed for mRNA (green), THOC2 (red) and nuclear DNA (blue). Scale bar, $10 \mu \mathrm{m}$. 


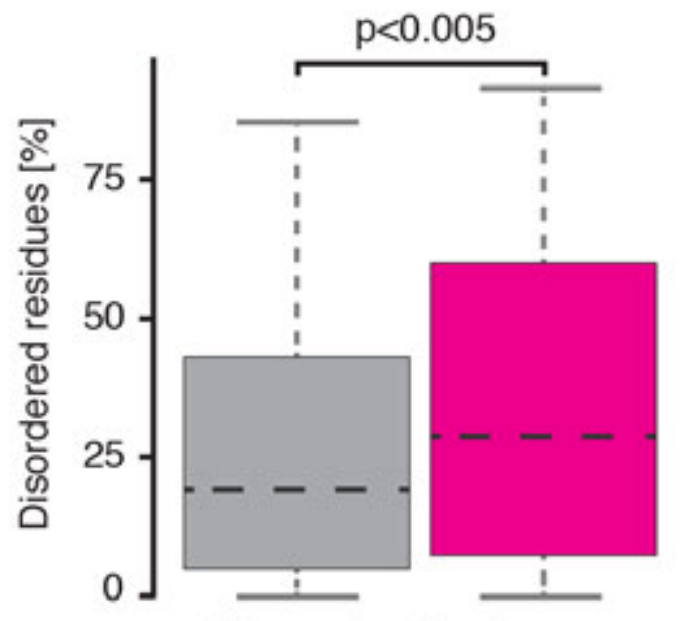

All proteins $\beta 17$ interactors

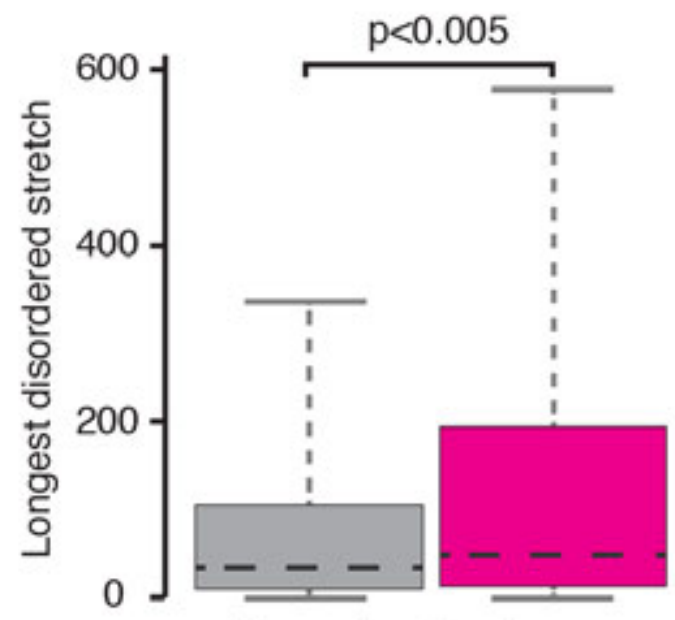

All proteins $\beta 17$ interactors

\section{Fig. S11}

Disorder analysis of $\boldsymbol{\beta 1 7}$ interactors. (A) Percentage of disordered residues and (B) longest disordered stretch, as predicted by IUPred, in interactor sequences, compared to the background proteins. Dashed horizontal line indicates the median, boxes indicate 25 th $/ 75$ th, and whisker caps 5 th/95th percentiles, respectively. P values are based on Mann-Whitney test. 


\section{Additional Data table S1 (separate file)}

Highly enriched annotations in the $\beta 17-G F P$ interactome in primary neurons. Results for the Fisher exact test on annotations of significant $\beta 17$ interactors. Listed are annotations (Gene Ontology Biological Process (GOBP), Cellular Component (GOCC), Molecular Function (GOMF), Kyoto Encyclopedia of Genes and Genomes (KEGG)) that were significantly (FDR $<2 \%$ ) enriched (enrichment factor $>2$ ). Categories are ordered according to enrichment, which is calculated with the following equation: [(\# proteins significantly enriched that belong to a certain category)*(\# all proteins)]/[(\# significantly enriched proteins)*(category size)]. The p-value is calculated based on a hypergeometric distribution.

\section{Additional Data table S2 (separate file)}

Annotations for individual $\beta 17$ interactors present in enriched annotation categories. Ratio of low complexity sequences to complex sequences based on SEG algorithms (43), and percentage of disordered regions and longest stretch of disordered residues as predicted by IUPred-L (46). The p-value was calculated with Welch's $t$ test (unequal variances). To determine significantly enriched proteins for $\beta 17$, we performed an S0 correction (emphasizing strength of enrichment) of 1 (42) and applied a permutation-based false discovery rate of $5 \%$ with PERSEUS. Proteins in each category are ordered alphabetically.

\section{Additional Data table S3 (separate file)}

Complete $\beta 17-G F P$ interactome in primary neurons (A) Label free quantification (MaxLFQ) (40) of interaction proteomes. (B) Processed MaxLFQ data with matched annotations and t-test statistics, which is used in outlier determination. Proteins that are significantly enriched (applying a permutation based false discovery cut off at $5 \%$, see Methods) are marked with "+” (C) Details of table headers in (A) and (B) 